\title{
The Golden Channel at a Neutrino Factory revisited: improved sensitivities from a Magnetised Iron Neutrino Detector
}

\author{
R. Bayes, A. Laing, and F.J.P. Soler \\ School of Physics \& Astronomy, University of Glasgow, Glasgow, UK.
}

A. Cervera Villanueva, J.J. Gómez Cadenas, P. Hernández, and J. Martín-Albo IFIC, CSIC $\&$ Universidad de Valencia, Valencia, Spain.

\section{J. Burguet-Castell}

Universitat de les Illes Balears, Spain.

\begin{abstract}
This paper describes the performance and sensitivity to neutrino mixing parameters of a Magnetised Iron Neutrino Detector (MIND) at a Neutrino Factory with a neutrino beam created from the decay of $10 \mathrm{GeV}$ muons. Specifically, it is concerned with the ability of such a detector to detect muons of the opposite sign to those stored (wrong-sign muons) while suppressing contamination of the signal from the interactions of other neutrino species in the beam. A new more realistic simulation and analysis, which improves the efficiency of this detector at low energies, has been
\end{abstract} developed using the GENIE neutrino event generator and the GEANT4 simulation toolkit. Low energy neutrino events down to $1 \mathrm{GeV}$ were selected, while reducing backgrounds to the $10^{-4}$ level. Signal efficiency plateaus of $\sim 60 \%$ for $\nu_{\mu}$ and $\sim 70 \%$ for $\bar{\nu}_{\mu}$ events were achieved starting at $\sim 5 \mathrm{GeV}$. Contamination from the $\nu_{\mu} \rightarrow \nu_{\tau}$ oscillation channel was studied for the first time and was found to be at the level between $1 \%$ and $4 \%$. Full response matrices are supplied for all the signal and background channels from $1 \mathrm{GeV}$ to $10 \mathrm{GeV}$. The sensitivity of an experiment involving a MIND detector of 100 ktonnes at $2000 \mathrm{~km}$ from the Neutrino Factory is calculated for the case of $\sin ^{2} 2 \theta_{13} \sim 10^{-1}$. For this value of $\theta_{13}$, the accuracy in the measurement of the $\mathrm{CP}$ violating phase is estimated to be $\Delta \delta_{C P} \sim 3^{\circ}-5^{\circ}$, depending on the value of $\delta_{C P}$, the $\mathrm{CP}$ coverage at $5 \sigma$ is $85 \%$ and the mass hierarchy would be determined with better than $5 \sigma$ level for all values of $\delta_{C P}$.

PACS numbers: 14.60.Ef,14.60.Pq,29.20.D-,29.40.-n,29.40.Mc 
Keywords: Neutrino Factory; Golden Channel; Magnetised Iron Neutrino Detector, MIND, $\mathrm{CP}$ violation 


\section{INTRODUCTION}

The Neutrino Factory, a new type of accelerator facility in which a neutrino beam is created from the decay of muons in flight in a storage ring, is perhaps the most promising facility design to resolve the problem of $\mathrm{CP}$ violation in the neutrino sector. The physics potential of this facility was first described by Geer [1]. The expected absolute flux and spectrum of neutrinos from such a facility can be calculated with smaller systematic errors than those associated with the beams of alternate facilities due to the ability to measure the muon beam flux and the highly accurate measurement of muon decay kinematics [2]. Since, in principle, both $\mu^{+}$and $\mu^{-}$can be created with the same systematic uncertainties on the flux, any oscillation channel can be studied with both neutrinos and antineutrinos, improving sensitivity to $\mathrm{CP}$ violation. Table $\mathrm{I}$ shows the oscillation channels that will contribute to the flux at any far site due to the decay of $\mu^{+}$.

TABLE I: Oscillation channels contributing to flux from the decay of $\mu^{+}$.

\begin{tabular}{c|c}
$\nu_{e}$ origin & $\bar{\nu}_{\mu}$ origin \\
\hline$\nu_{e} \rightarrow \nu_{e}\left(\nu_{e}\right.$ disappearance channel $)$ & $\bar{\nu}_{\mu} \rightarrow \bar{\nu}_{\mu}\left(\bar{\nu}_{\mu}\right.$ disappearance channel $)$ \\
$\nu_{e} \rightarrow \nu_{\mu}$ (Golden channel $)$ & $\bar{\nu}_{\mu} \rightarrow \bar{\nu}_{\tau}$ (Dominant oscillation) \\
$\nu_{e} \rightarrow \nu_{\tau}$ (Silver channel) & $\bar{\nu}_{\mu} \rightarrow \bar{\nu}_{e}$ (Platinum channel)
\end{tabular}

The sub-dominant $\nu_{e} \rightarrow \nu_{\mu}$ oscillation [3] was identified as the most promising channel to explore CP violation at a Neutrino Factory. The charged current interactions of the "Golden Channel" $\nu_{\mu}$ produce muons of the opposite charge to those stored in the storage ring (wrong-sign muons) and these can be detected with a large magnetised iron detector [4]. The original analyses were carried out assuming a Neutrino Factory storing $50 \mathrm{GeV}$ muons and, as such, were optimised for high energy using a detector with $4 \mathrm{~cm}$ thick iron plates and $1 \mathrm{~cm}$ scintillator planes. However, subsequent phenomenological studies carried out as part of the International Scoping Study (ISS) for future neutrino facilities [5, 6] favoured a stored muon energy of $25 \mathrm{GeV}$ and showed the importance of neutrinos with energies below $5 \mathrm{GeV}$. The Magnetised Iron Neutrino Detector (MIND) is a large scale iron and scintillator sampling calorimeter, similar to MINOS [7], which was re-optimized from the 
original studies motivated by these findings [8-10]. The performance obtained indicated that the combination of two Magnetised Iron Neutrino Detectors at $4000 \mathrm{~km}$ and $7500 \mathrm{~km}$ would give optimum sensitivity to the mixing parameters [11].

The studies of MIND mentioned above evaluated the performance of the detector using deep inelastic scattering events only, with a simplified simulation, reconstruction and kinematic analysis. The performance needed to be evaluated and improved using a full simulation and analysis of all physical processes. As part of the International Design Study for a Neutrino Factory [12, 13], a software framework to perform these studies has been developed. Pattern recognition and analysis algorithms were developed and first applied to data generated using the same simulation as was used in the ISS studies. The development of the algorithm and the results of its application were described in [14] and [15], where it was shown that under these conditions the efficiency and background could be maintained at a similar level to that achieved in the ISS studies. This paper introduces the full spectrum of possible neutrino interactions generated using the neutrino event generator GENIE [16] and a comparison with another event generator, NUANCE [17]. These interactions were tracked through a new GEANT4 simulation [18, 19] with full hadron shower development and a new detector digitisation not present in previous studies. The events were then subject to the pattern recognition algorithm presented in [15], reoptimised for the new simulation. Finally a likelihood based analysis was used to further suppress backgrounds. A preliminary version of this analysis using the NUANCE package has been published in the Interim Design Report of the IDS-NF [20]. This paper includes the full GENIE simulation, a comparison to NUANCE, an estimate of systematic errors, and sensitivity calculations for $\theta_{13}$, the neutrino mass hierarchy (sign of $\Delta m_{13}^{2}=m_{1}^{2}-m_{3}^{2}$ ) and the CP violating phase $\delta_{C P}$.

Recent results from the reactor experiments Daya Bay, RENO and Double Chooz [21 23], as well as evidence from T2K [24] and MINOS [25], have demonstrated that the value of $\theta_{13}$ is large (with a combined average of $\sin ^{2} 2 \theta_{13}=0.097 \pm 0.012$ ). These results increase the likelihood of a discovery of CP violation and the determination of the mass hierarchy in neutrinos. It was shown in the Interim Design Report of the IDS-NF [20] that at a value of $\sin ^{2} 2 \theta_{13} \sim 0.1$ the optimum Neutrino Factory configuration is achieved with a muon energy of $10 \mathrm{GeV}$ and with a far detector at a distance of $2000 \mathrm{~km}$. While the Neutrino Factory was designed to discover $\mathrm{CP}$ violation for a large range of values of $\theta_{13}$ (down to values of $\sin ^{2} 2 \theta_{13} \sim 10^{-4}$ ), it will be shown in this paper that it also offers the best chance to discover 
$\mathrm{CP}$ violation and the mass hierarchy at large values of $\theta_{13}$, regardless of whether $\Delta m_{13}^{2}$ is positive or negative (inverted or normal mass hierarchy).

This paper is organised as follows. Section II introduces the relevant backgrounds and contaminations of the golden signal and describes the required suppressions. Section III describes the simulation tools and gives a description of MIND and the assumptions still made for this study. The analysis is described in Section IV, with a detailed demonstration of all variables and functions used to identify signal from background. The results from this analysis, including signal efficiencies, background rejection capabilities and performance to the $\nu_{\mu} \rightarrow \nu_{\tau}$ oscillation signal, are presented in Section $\mathrm{V}$, A discussion of some of the systematic errors of the analysis is described in Section VI. Finally, full sensitivity to $\theta_{13}$, $\delta_{C P}$ and the neutrino mass hierarchy will be presented in Section VIII. Response (migration) matrices of this detector system for all signal and background will be shown in the Appendix.

\section{SOURCES OF IMPURITY IN THE GOLDEN SAMPLE}

The primary sources of background to the wrong-sign muon search come from the Charged Current (CC) and Neutral Current (NC) interactions of the non-oscillating neutrinos present in the beam. Specifically, the CC interactions of $\nu_{\mu}\left(\bar{\nu}_{\mu}\right)$ being reconstructed as $\bar{\nu}_{\mu}\left(\nu_{\mu}\right)$, NC from all neutrino types in the beam being reconsructed as $\bar{\nu}_{\mu}\left(\nu_{\mu}\right)$ and the CC interactions of $\bar{\nu}_{e}\left(\nu_{e}\right)$ being reconstructed as $\bar{\nu}_{\mu}\left(\nu_{\mu}\right)$. Since these interactions are in far greater abundance than those of the signal channel and contain little or no discernible information about the key parameters $\theta_{13}$ and $\delta_{C P}$, they must be suppressed sufficiently so that the statistical error on the background is smaller than the expected signal level. This corresponds to a suppression of at least $10^{-3}$ for each channel in the signal region.

In addition to the golden channel appearance oscillation there are three other appearance channels that will introduce neutrinos to the flux incident on the far detectors (shown in table I). The dominant oscillation, $\nu_{\mu}\left(\bar{\nu}_{\mu}\right) \rightarrow \nu_{\tau}\left(\bar{\nu}_{\tau}\right)$, which must be considered when fitting for the $\nu_{\mu}\left(\bar{\nu}_{\mu}\right)$ disappearance signal, should not pose a problem for fitting the golden channel since, at large $\theta_{13}$, the dependence of this channel on this mixing angle is very small and the interaction would have to be reconstructed with the opposite charge to that of the true primary lepton. See [26] for a detailed discussion of tau contamination in the disappearance channel. The platinum channel, $\nu_{\mu}\left(\bar{\nu}_{\mu}\right) \rightarrow \nu_{e}\left(\bar{\nu}_{e}\right)$, should pose no problem 
since the number of interactions should be similar to that produced by the golden channel and $\nu_{e}$ interactions produce a penetrating muon-like track in only a small fraction of cases. However, the silver channel oscillation, $\nu_{e}\left(\bar{\nu}_{e}\right) \rightarrow \nu_{\tau}\left(\bar{\nu}_{\tau}\right)$, would be expected to contribute a similar amount of $\nu_{\tau}$ to the flux as the golden channel does to $\nu_{\mu}$, and since the primary $\tau$ decays with $\mathrm{a} \sim 17.65 \%$ probablility via channels containing muons, a significant proportion of these interactions would be expected to pass the analysis cuts. As discussed in [27], fitting the observed spectrum without accounting for the presence of these $\nu_{\tau}$ interactions leads to significantly reduced accuracy in the fits. However, since this oscillation contains complimentary information about both $\theta_{13}$ and $\delta_{C P}$, handling this 'contamination' correctly has the potential to perhaps improve the fit accuracy compared to using an analysis which attempts to remove it from the sample.

\section{SIMULATION AND RECONSTRUCTION OF MIND}

In previous studies [4, 10, 15], only deep inelastic scattering (DIS) events generated with LEPTO 6.1 [28] were considered. However, at energies below $5 \mathrm{GeV}$ there are large contributions from quasi-elastic (QE), single pion production $(1 \pi)$ and other resonant production (RES) events. QE and $1 \pi$ events are expected to exhibit lower multiplicity in the detector output, which makes candidate muon reconstruction simpler. This should improve reconstruction efficiency in low energy CC interactions but also potentially increase low energy backgrounds, particularly from NC $1 \pi$ interactions. Other nuclear resonant events, producing two or three pions, as well as diffractive and coherent production, have much smaller contributions. Moreover, the presence of QE interactions allows for the calculation of neutrino energy without hadron shower reconstruction, improving neutrino energy resolution.

\section{A. Neutrino event generation and detector simulation}

Generation of all types of interaction was performed using the GENIE framework [17]. The exclusive event samples generated by GENIE are shown in figure 1, where 'other' interactions include the resonant, coherent and diffractive processes other than single pion production. The relative rates below $1 \mathrm{GeV}$ are included for completeness, but will have negligible effect at a Neutrino Factory. GENIE also includes a treatment to simulate the 
effect of re-interaction within the participant nucleon, which is particularly important for low energy interactions in high- $Z$ targets such as iron.

A new simulation of MIND using the GEANT4 toolkit [19] (G4MIND) was developed to provide flexibility to the definition of the geometry, to carry out full hadron shower development and to perform a proper digitisation of the events. This allows optimisation of all aspects of the detector, such as the dimensions and spacing of all scintillator and iron pieces, external dimensions of the detector and detector readout considerations.

The detector transverse dimensions ( $x$ and $y$ axes) and length in the beam direction $(z$ axis), transverse to the detector face, are controlled from a parameter file. A fiducial cross section of $14 \mathrm{~m} \times 14 \mathrm{~m}$, including $3 \mathrm{~cm}$ of iron for every $2 \mathrm{~cm}$ of polystyrene extruded plastic scintillator (1 cm of scintillator per view), was assumed. A constant magnetic field of $1 \mathrm{~T}$ is oriented in the positive $y$ direction throughout the detector volume. Events generated for iron and scintillator nuclei are selected according to their relative weights in the detector and the resultant particles are tracked from a vertex randomly positioned in three dimensions within a randomly selected piece of the appropriate material. Physics processes are modelled using the QGSP_BERT physics lists provided by GEANT4 [29].

Secondary particles are required to travel at least $30 \mathrm{~mm}$ from their production point or to cross a material boundary between the detector sub-volumes to have their trajectory fully tracked. Generally, particles are only tracked down to a kinetic energy of $100 \mathrm{MeV}$. However, gammas and muons are excluded from this cut. The end-point of a muon track is important for muon pattern recognition.

A simplified digitisation model was considered for this simulation. Two-dimensional boxes - termed voxels - represent view-matched $x$ and $y$ readout positions. Any deposit which falls within a voxel has its energy deposit added to the voxel total raw energy deposit. The thickness of two centimetres of scintillator per plane assumes $1 \mathrm{~cm}$ per view. Voxels with edge lengths of $3.5 \mathrm{~cm}$ were chosen to match the required point resolution of $1 \mathrm{~cm}$ $(3.5 / \sqrt{12})$, assuming a uniform hit distribution along the width of the scintillator bar. The response of the scintillator bars is derived from the raw energy deposit in each voxel, read out using wavelength shifting (WLS) fibres with attenuation length $\lambda=5 \mathrm{~m}$, as reported by the MINERvA collaboration [30]. Assuming that approximately half of the energy will come from each view, the deposit is halved and the remaining energy at each edge in $x$ and $y$ is calculated. This energy is then smeared according to a Gaussian with $\sigma / E=6 \%$ to 


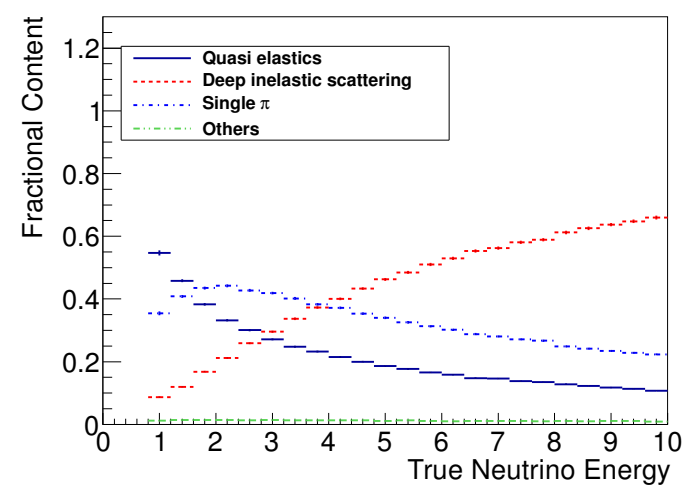

(a) $\bar{\nu}_{\mu} \mathrm{CC}$

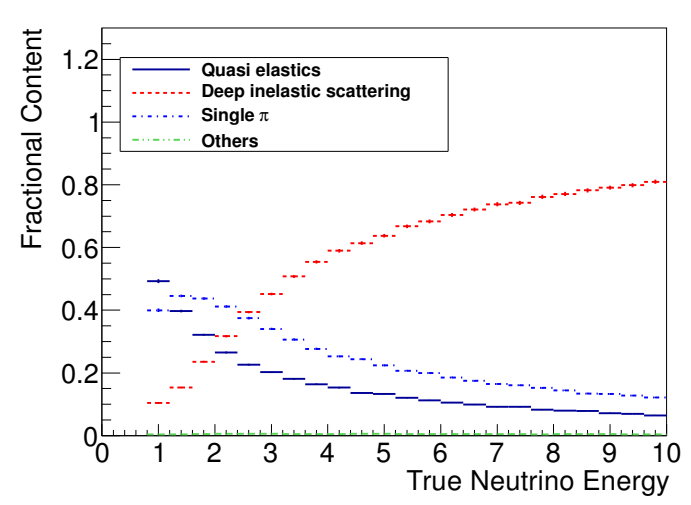

(c) $\nu_{\mu} \mathrm{CC}$

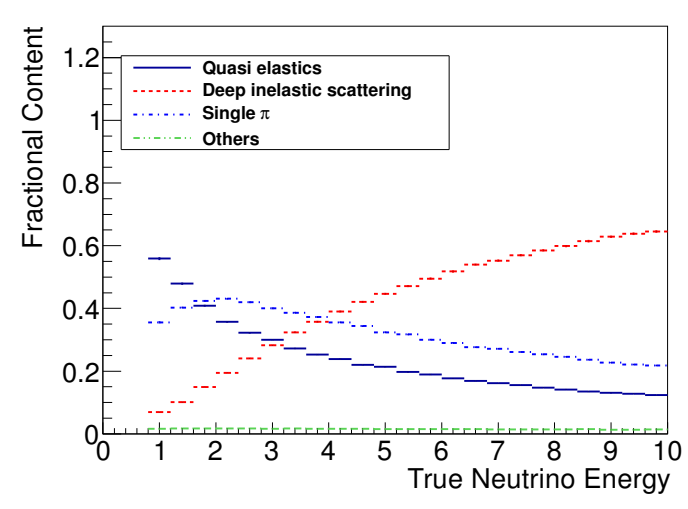

(e) $\bar{\nu}_{\mu} \mathrm{NC}$

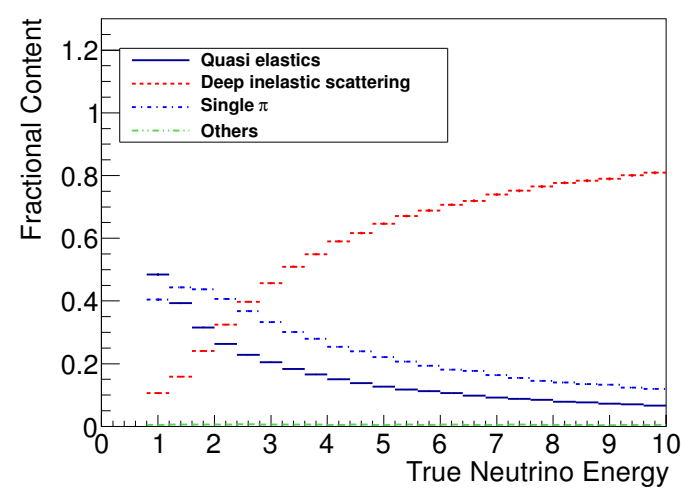

(b) $\nu_{e} \mathrm{CC}$

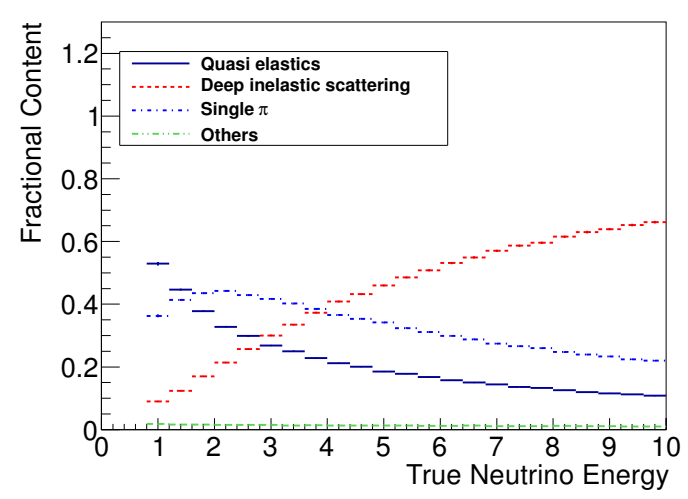

(d) $\bar{\nu}_{e} \mathrm{CC}$

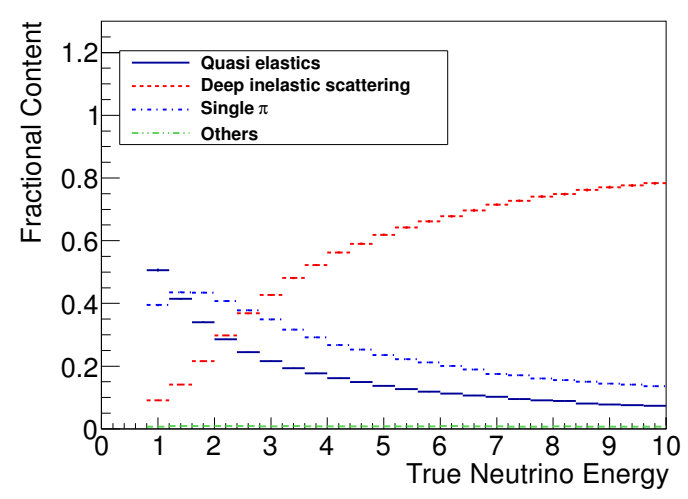

(f) $\nu_{\mu} \mathrm{NC}$

FIG. 1: Proportion of total number of interactions of different $\nu$ interaction processes for events generated using GENIE and passed to the G4MIND simulation. 

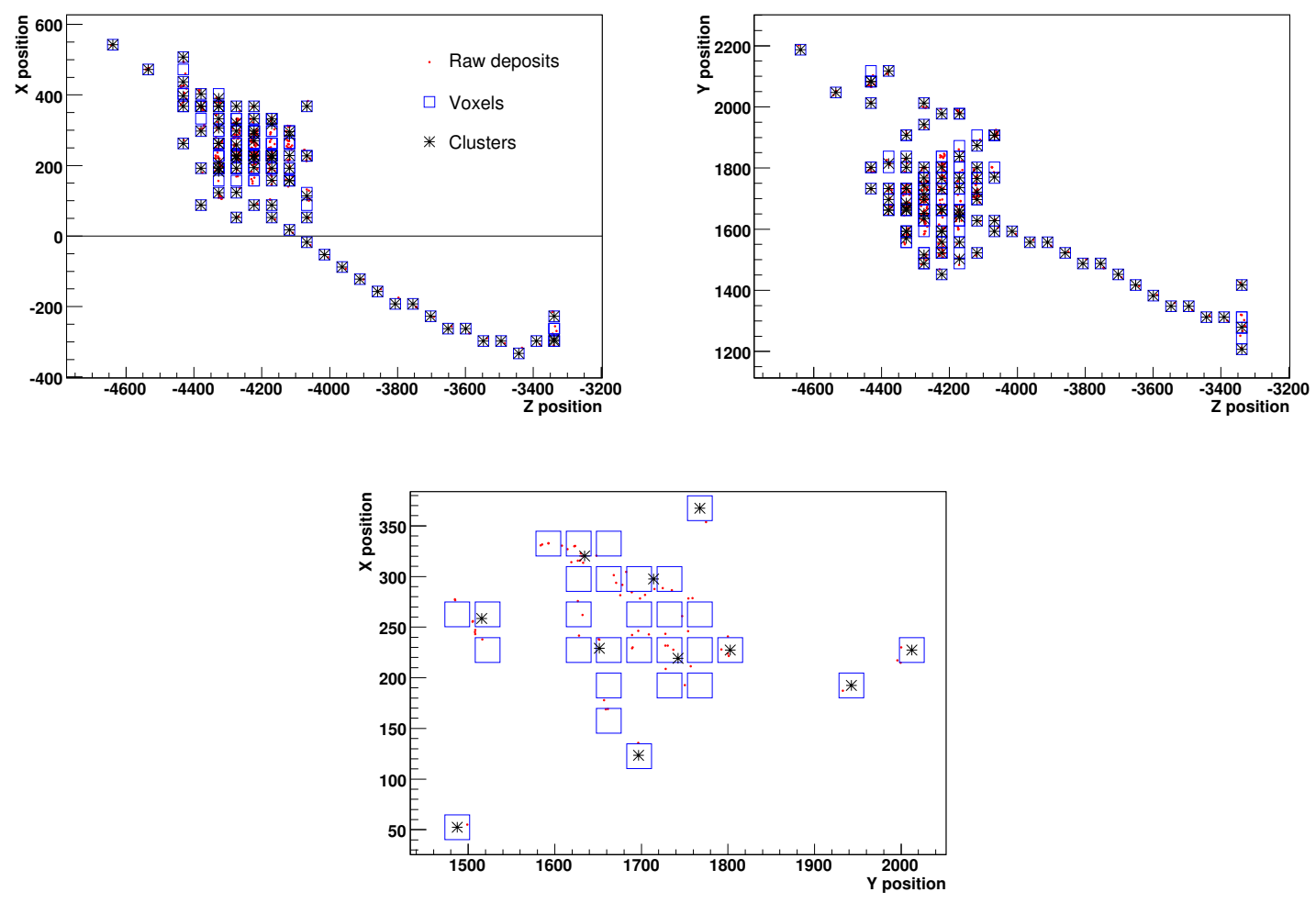

FIG. 2: The digitisation and voxel clustering of an example event: (top left) bending plane view, (top right) non bending plane, (bottom) an individual scintillator plane. The individual hits are small dots (in red), the blue squares are the voxels and the black asterisks represent the centroid positions of the clusters.

represent the response of the electronics and then recombined into $x, y$ and total $=x+y$ energy deposit per voxel. An output wavelength of $525 \mathrm{~nm}$, a photo-detector quantum efficiency of $\sim 30 \%$ and a threshold of 4.7 photo electrons (pe) per view (as in MINOS [7]) were assumed. Any voxel in which the two views do not make this threshold is cut. If only one view is above threshold, then only the view below the cut is excluded (see section III B). The digitisation of an example event is shown in figure 2.

\section{B. Event reconstruction}

The reconstruction package was described in detail in [15]. We present here an update of the reconstruction based on the MIND simulation generated using GENIE and GEANT4.

Many traversing particles, particularly hadrons, deposit energy in more than one voxel. 
Forming clusters of adjacent voxels reduces event complexity and can improve pattern recognition in the region of the hadron shower. The clustering algorithm is invoked at the start of each event. The voxels of every plane in which energy has been deposited are considered in sequence. Where an active voxel is in contact with no other active voxel, this voxel becomes a cluster. If there are adjacent voxels, the voxel with the largest total deposit (at scintillator edge) is sought and all active voxels in the surrounding $3 \times 3$ area are considered part of the cluster. Adjacent deposits that do not fall into this area are considered separate. The cluster position is calculated independently in the $x$ and $y$ views as the energy-weighted sum of the individual voxels. One voxel, two voxel and three voxel clusters were found to have position resolutions of $9.4 \mathrm{~mm}, 8.0 \mathrm{~mm}$ and $7.2 \mathrm{~mm}$, respectively. The improved resolution due to clusters with multiple voxels is due to the charge sharing between voxels. The clusters formed from the hit voxels of an event are then passed to the reconstruction algorithm.

The separation of candidate muons from hadronic activity is achieved using two methods: a Kalman filter algorithm provided by RecPack [31] and a cellular automaton method (based on [32]), both algorithms are described in detail in [15. The Kalman filter method requires a section of at least five planes where only one cluster is present in the highest $z$ region of the event that is associated with particle tracks. Between $85 \%$ and $95 \%$ of $\nu_{\mu}\left(\bar{\nu}_{\mu}\right) \mathrm{CC}$ interactions and $\sim 2.5 \%$ of $\mathrm{NC}$ interactions fall into this category. This section is used to form a seed, which is projected back through the high occupancy planes using a helix model. Events which do not have such a section (generally high $Q^{2}$ or low neutrino energy events) are subject to the cellular automaton which tests a number of possible tracks to find a potential muon candidate. Between $5 \%$ and $13 \%$ of $\nu_{\mu}\left(\bar{\nu}_{\mu}\right)$ CC interactions and $\sim 83 \%$ of $\mathrm{NC}$ are presented to the cellular automaton for consideration. NC events produce a candidate muon which is successfully fitted as such in $\sim 60 \%$ of cases sent to the Kalman filter. Of the $\sim 28 \%$ $\nu_{\mu}\left(\bar{\nu}_{\mu}\right)$ CC events sent to the cellular automaton method $99 \%$ of $\nu_{\mu}$ and $45 \%$ of $\bar{\nu}_{\mu}$ are successfully fitted.

Compared to the method used and described in detail in [15], an additional step has been added to the reconstruction method to take into account that fully-contained muons (particularly $\mu^{-}$) can have additional deposits at their endpoint due to captures on nuclei or due to decays. Long, well defined tracks can be rejected if there is added energy deposited at the muon end point, since this can be interpreted as hadronic activity and rejected by the Kalman filter method, thereby confusing the track finding algorithm of the cellular automa- 

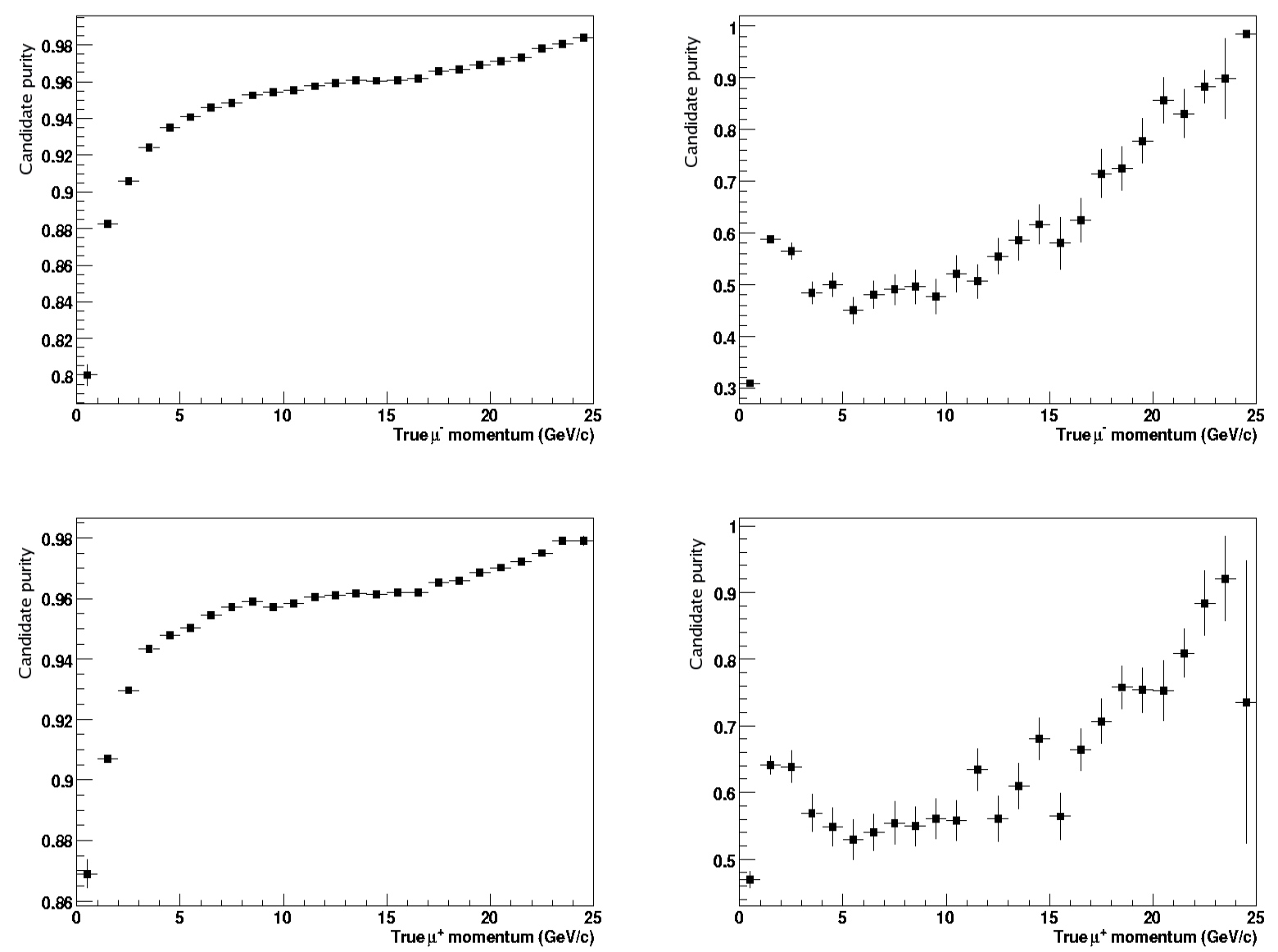

FIG. 3: Muon candidate hit purity for $\nu_{\mu} \mathrm{CC}$ (top) and $\bar{\nu}_{\mu} \mathrm{CC}$ (bottom) interactions extracted using (left) Kalman filter method and (right) cellular automaton method.

ton. Therefore, after sorting clusters into increasing $z$ position, an additional algorithm is used to identify such activity and extract the track section for seeding and projection. The details of this algorithm can be found in [33], but it relies on identifying isolated muon-like hits at the end of a track and removing the high activity region in the choice of seeds to perform the track fit.

The complete pattern-recognition chain using these algorithms leads to candidate purity (fraction of candidate hits of true muon origin) for $\nu_{\mu}\left(\bar{\nu}_{\mu}\right) \mathrm{CC}$ events as shown in figure 3 . A cluster is considered to be of muon origin if greater than $80 \%$ of the raw deposits contained within the cluster were recorded as muon deposits.

Fitting of the candidates proceeds using a Kalman filter to fit a helix to the candidate, using an initial seed estimated by a quartic fit, and then refitting any successes. Projecting 

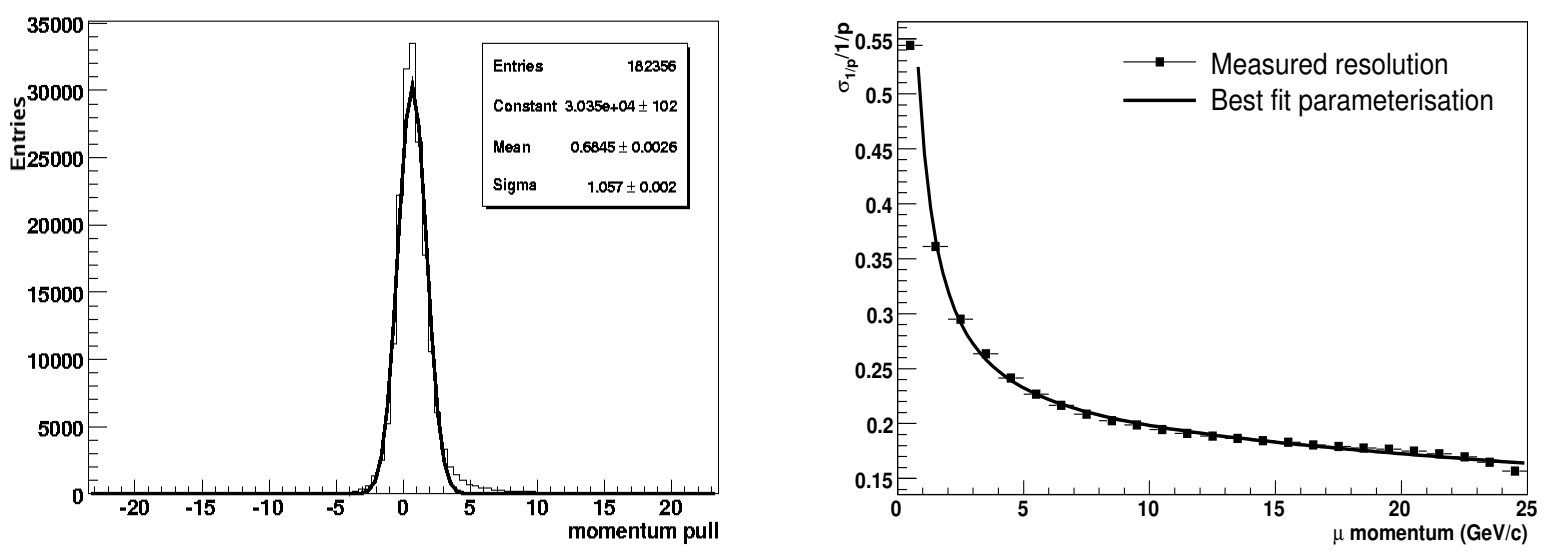

FIG. 4: Pull on the reconstructed momentum (the difference between the true and reconstructed momentum divided by the measured error) (left) and momentum resolution (right).

successful trajectories back to the true vertex $z$ position, the quality of the fitter can be estimated by comparing to the pull distribution of the reconstructed momentum, defined as the difference in true and reconstructed momentum, divided by the measured error in the momentum from the fit (see figure 4). The error in the momentum pull is as expected, but the mean pull has a bias $(+0.68)$, due to an incomplete energy-loss model in the Kalman filter. This small bias is taken into account in the migration matrices derived for this analysis (see Appendix). Further improvements to the energy-loss model within Recpack are being carried out and should reduce any residual bias. An empirical parametrisation of the momentum resolution is also shown in figure 4, which can be written as follows:

$$
\frac{\sigma_{1 / p}}{1 / p}=0.18+\frac{0.28}{p(G e V)}-1.17 \times 10^{-3} p(G e V)
$$

Neutrino energy is generally reconstructed as the sum of the muon and hadronic energies, with hadronic reconstruction currently performed using a smear on the true quantities as described in ref. [15]. The reconstruction of the hadronic energy $E_{\text {had }}$ assumes a resolution $\delta E_{\text {had }}$ from the MINOS CalDet testbeam [7, 34]:

$$
\frac{\delta E_{\text {had }}}{E_{\text {had }}}=\frac{0.55}{\sqrt{E_{\text {had }}}} \oplus 0.03
$$

The hadronic shower direction vector was also smeared according to the angular resolution 
found by the Monolith test-beam [35]:

$$
\delta \theta_{\text {had }}=\frac{10.4}{\sqrt{E_{\text {had }}}} \oplus \frac{10.1}{E_{\text {had }}} .
$$

In the case of $\mathrm{QE}$ interactions, where there is no hadronic jet, the neutrino energy reconstruction was carried out using the formula:

$$
E_{\nu}=\frac{m_{N} E_{\mu}+\frac{1}{2}\left(m_{N^{\prime}}^{2}-m_{\mu}^{2}-m_{N}^{2}\right)}{m_{N}-E_{\mu}+\left|p_{\mu}\right| \cos \vartheta}
$$

where $\vartheta$ is the angle between the muon momentum vector and the beam direction, $m_{N}$ is the mass of the initial state nucleon, and $m_{N^{\prime}}$ is the mass of the outgoing nucleon for the interactions $\nu_{\mu}+n \rightarrow \mu^{-}+p$ and $\bar{\nu}_{\mu}+p \rightarrow \mu^{+}+n$ (see for example [36]). The current algorithm only uses this formula for the case of events consisting of a single unaccompanied track, however, its use could be extended by selecting QE interactions using their distribution in $\vartheta$ and their event-plane occupancy among other parameters. Should the use of equation 4 result in a negative value for the energy, it is recalculated as the total energy of a muon with its reconstructed momentum.

\section{ANALYSIS OF POTENTIAL SIGNAL AND BACKGROUND}

There are four principal sources of background to the wrong sign muon search: charge mis-identification of the primary muon in $\nu_{\mu}$ charged current (CC) interactions, wrong sign muons from hadron decay in $\bar{\nu}_{\mu} \mathrm{CC}$ events, neutral current (NC) from all species and $\nu_{e} \mathrm{CC}$ events wrongly identified as $\nu_{\mu}$ CC. Typically, a $\nu_{\mu}$ charged current event has greater length in the beam direction than a $\mathrm{NC}$ or $\nu_{e} \mathrm{CC}$ event, due to the penetrating muon. Any muons produced from the decay of primary interaction hadrons will tend to be less isolated from other hadronic activity. Additionally, the $\nu_{e}$ spectrum at a Neutrino Factory has a lower average energy than the $\nu_{\mu}$ spectrum which results in reduced probability of producing a high energy particle in the interaction.

The previous general principles are used to define a series of offline cuts that reject the dominant background while maintaining good signal efficiency. These can be organised in three categories: 1) track quality cuts; 2) charged current selection cut; and 3) kinematic cuts. We will describe these cuts in detail in sub-sections IV A, IV B and IV C. A summary of the performance of each of the cuts on signal and background will be presented in sub-

section IVD and table II. 

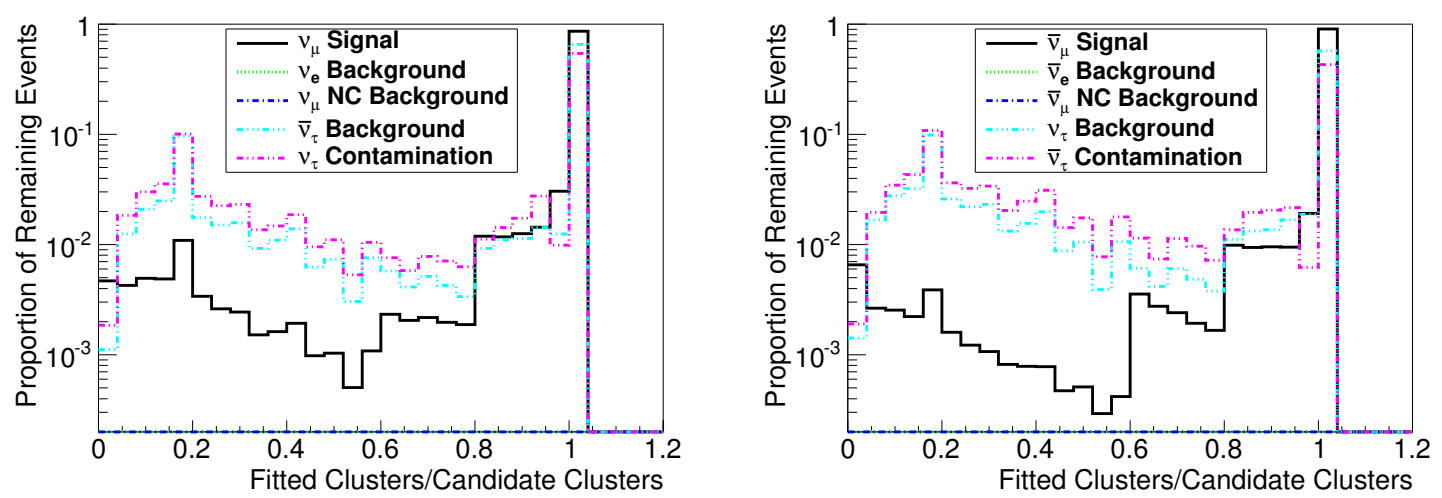

FIG. 5: Distribution of the proportion of clusters fitted in the trajectory for $\nu_{\mu}$ appearance (left) and $\bar{\nu}_{\mu}$ appearance (right), normalised to total remaining events individually for each interaction type.

\section{A. Track quality cuts}

The quality of the reconstruction and the error on the momentum parameter of the Kalman filter are powerful handles in the rejection of backgrounds. We commence by imposing the reconstruction criteria from the previous section to guarantee fully reconstructed neutrino events. We then proceed to impose a fiducial cut requiring that $z 1$, which is the cluster with the lowest $z$ in the candidate, be at least $2 \mathrm{~m}$ from the end of the detector $\left(z 1-z_{\text {end }} \geq 2000 \mathrm{~mm}\right)$, to reduce the mis-identification of candidates originating at high z. Additionally, a maximum value for the reconstructed muon momentum is imposed at $16 \mathrm{GeV}$ to improve energy resolution and remove backgrounds caused by very straight particles, which confuse the fitter.

Tracks dominated by multiple scattering or incorporating deposits made by particles not left by a muon can contribute significantly to backgrounds. However, these tracks will tend to be fitted only partially or with a larger error on the momentum variables. As such, cuts on these variables can be used to reduce the effect of these backgrounds. The distribution of the ratio of the candidate clusters which are fitted with respect to the total number of candidate clusters for signal and background is shown in figure 5. Accepting only those events in which a candidate has more than $60 \%$ of its clusters fitted reduces the background levels.

Further reduction is achieved by performing a cut related to the relative error in the mo- 

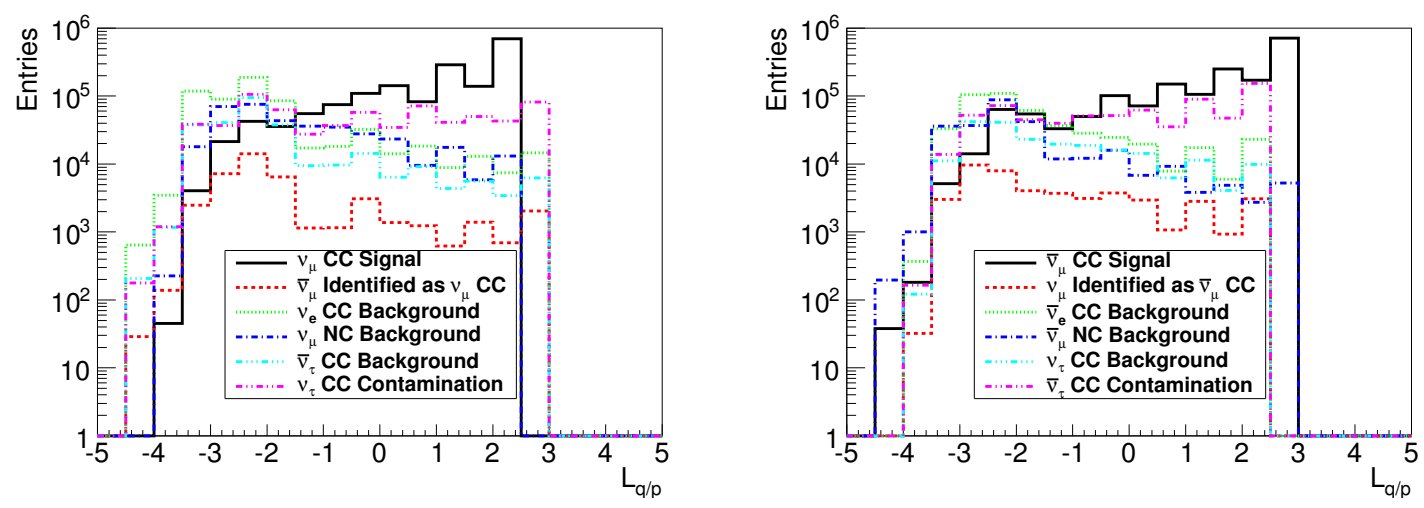

FIG. 6: Log likelihood distribution $\left(\mathcal{L}_{q / p}\right)$ to separate wrong-sign muons for signal and background for $\nu_{\mu}$ (left) and $\bar{\nu}_{\mu}$ (right) appearance experiments.

mentum of the candidate muon $\frac{\sigma_{q / p}}{q / p}$, where $q$ is the charge of the muon and $p$ its momentum. A log-likelihood distribution $\mathcal{L}_{q / p}$ based on the ratio of $\frac{\sigma_{q / p}}{q / p}$ for both signal and background is shown in figure6. The signal events are selected as those with a log-likelihood parameter $\mathcal{L}_{\sigma / p}>-0.5$.

After the preceding cuts there remain some background events which exhibit little bending due to the magnetic field or are reconstructed with relatively high momentum despite being relatively short tracks (see figure 7, right) as a result of high levels of multiple scattering. As can be seen in figure 7, left, removing short events in which the end point is displaced in the bending plane by an amount that is relatively small compared to the displacement in the lateral view (disp $X /$ disp $Z$ ) effectively reduces background. Events are accepted if they meet the conditions described in equations 5 and 6 , illustrated by the red lines in figure 7 .

$$
\begin{aligned}
& \frac{\operatorname{disp} X}{\operatorname{disp} Z}>0.18-0.0026 \cdot N_{h} ; \text { and } \\
& \operatorname{disp} Z>6000 \mathrm{~mm} \text { or } p_{\mu} \leq 3 \cdot \operatorname{disp} Z
\end{aligned}
$$

where $N_{h}$ is the number of clusters in the candidate, disp $Z$ is in units of $\mathrm{mm}$, and $p_{\mu}$ in units of $\mathrm{MeV} / \mathrm{c}$.

The final quality cut involves fitting to a parabola the candidate's projection onto the bending plane. In the current simulation a negatively-charged muon bends upwards, so that for a parabola defined as $a+b z+c z^{2}$ the parameter $c$ would be positive and the charge of the muon is $Q_{\text {par }}=-\operatorname{sign}(c)$. If the charge fitted is opposite to that found by the Kalman 

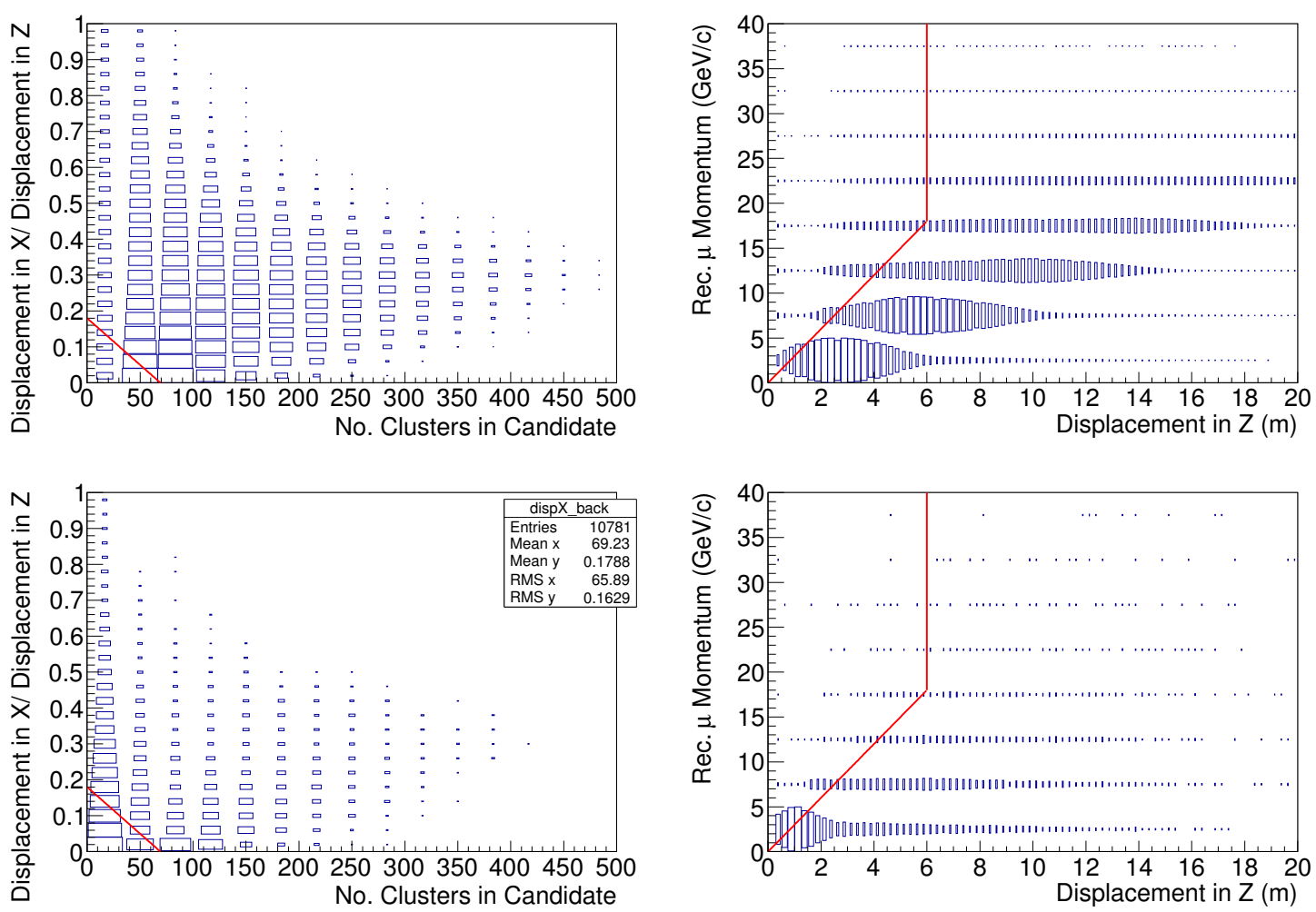

FIG. 7: Distributions of displacement and momentum with cut levels: (top left) relative displacement in the bending plane to the $z$ direction against candidate hits for signal events, (top right) reconstructed momentum against displacement in $z$ for signal events and (bottom) as top for $\nu_{\mu}\left(\bar{\nu}_{\mu}\right)$ CC backgrounds. The red lines represent the cuts from equations 5 and 6 .

filter, the quality of the fit is assessed using the variable:

$$
q p_{p a r}=\left\{\begin{array}{c}
\left|\frac{\sigma_{c}}{c}\right|, \text { if } Q_{p a r}=Q_{k a l} \\
-\left|\frac{\sigma_{c}}{c}\right|, \text { if } Q_{p a r}=-Q_{k a l}
\end{array}\right.
$$

where $Q_{k a l}$ is the charge fitted by the Kalman filter fit. Defining the parameter in this way ensures that the cut is independent of the initial fitted charge. Events with no charge change $\left(q p_{\text {par }}>0.0\right)$ are accepted as signal. Additionally, those fitted badly with a charge change $\left(q p_{\text {par }}<-1.0\right)$ are also accepted. In this way, background events which have remained in the sample due to local variations affecting the Kalman fitter can be removed without rejecting viable events in which the Kalman fitter ignored a section after a high angle scatter. The 

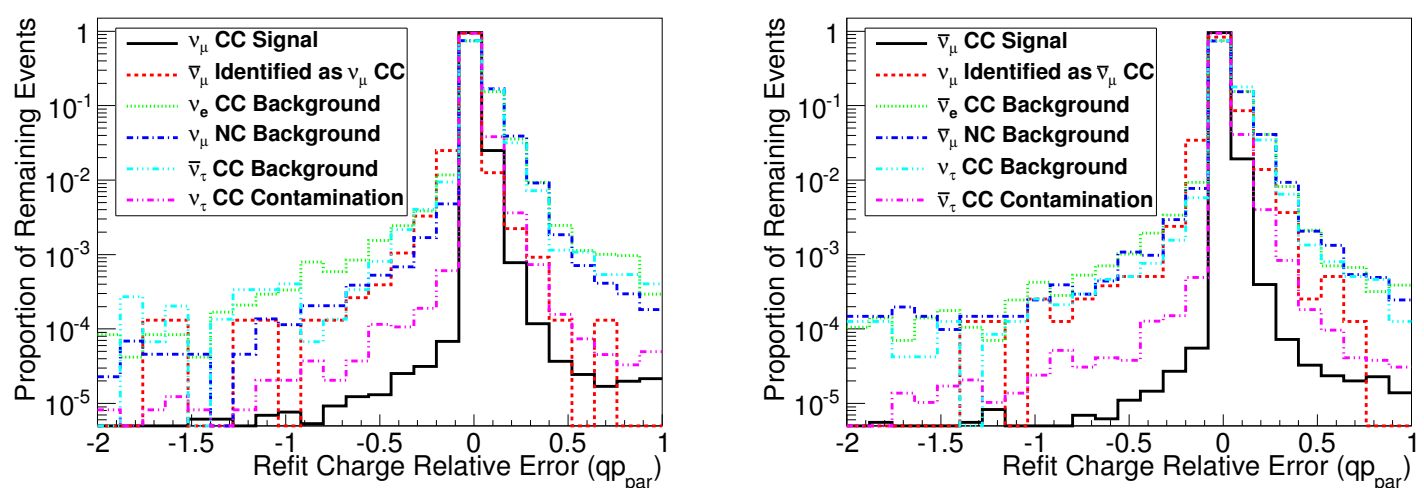

FIG. 8: Distribution of the $q p_{\text {par }}$ variable, with the region where the parameter is $<0$ representing those candidates fitted with charge opposite to the initial Kalman filter for $\nu_{\mu}$

(left) and $\bar{\nu}_{\mu}$ appearance experiments. The distributions are normalised to the total remaining events, individually, for each interaction type.

distribution of $q p_{p a r}$ is shown in figure 8 .

\section{B. Charged current selection}

Selection of charged currents and rejection of neutral current events is most efficiently performed by exploiting the propertiy that $\nu_{\mu} \mathrm{CC}$ events tend to have greater length in $z$ than NC events, since a true muon only interacts electromagnetically where a pion or kaon of similar momentum can interact via the strong force and will tend to stop after a shorter distance. Hence, the number of hits, $l_{h i t}$, was used to generate Probability Density Functions (PDF) for charged and neutral current events (see figure 9). One can see that the NC events have fewer reconstructed clusters than the equivalent $\nu_{\mu} \mathrm{CC}$ events. For the event selection, candidates with greater than 150 clusters are considered signal, otherwise, the log likelihood rejection parameter:

$$
\mathcal{L}_{1}=\log \left(\frac{l_{\text {hit }}^{C C}}{l_{\text {hit }}^{N C}}\right) ;
$$

is used, which is shown in figure 10, Allowing only those candidates where the log parameter is $\mathcal{L}_{1}>1.0$ to remain in the sample ensures that the sample is pure. This analysis is similar, but simpler, than that employed by MINOS [37]. The effect of the CC event selection is to reduce the background by one order of magnitude (see table II while having minimal effect 


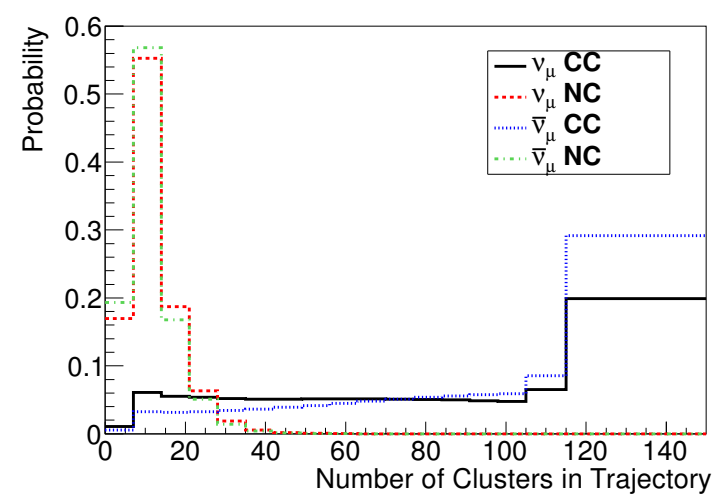

FIG. 9: Distribution of the number of fit clusters in candidate track used to calculate the log likelihood based charged current selection.

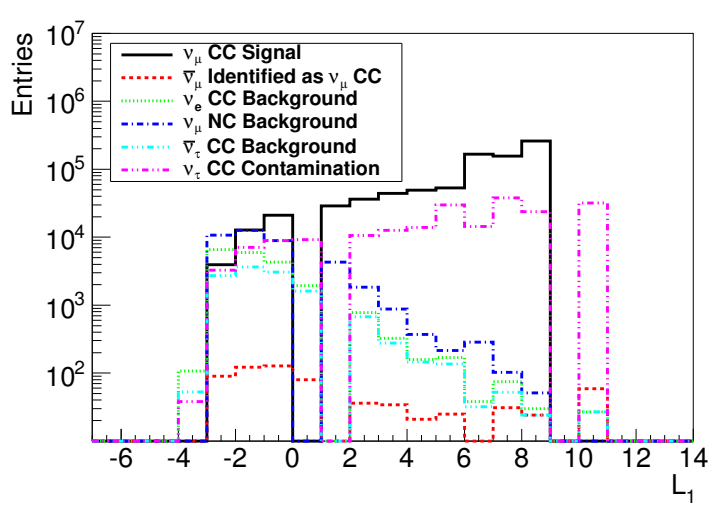

(a) Distribution for $\nu_{\mu}$ detection

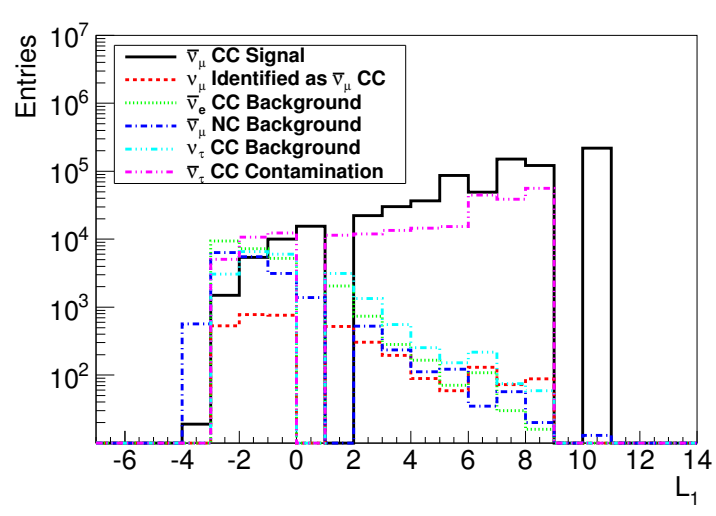

(b) Distribution for $\bar{\nu}_{\mu}$ detection

FIG. 10: Distribution of $\mathcal{L}_{1}$ likelihood ratios used to reject NC and other background signals.

on the signal efficiency.

\section{Kinematic cuts}

Kinematic cuts based on the momentum and isolation of the candidate, in relation to the reconstructed energy of the event $E_{r e c}$, can be used to reduce backgrounds from hadron decays. The isolation of the candidate muon is described by the variable $Q_{t}=p_{\mu} \sin ^{2} \theta$, where $\theta$ is the angle between the muon candidate and the hadronic-jet vector. The muon from a true $\mathrm{CC}$ event is generally isolated from the hadronic jet so, on average, the $Q_{t}$ is larger for $\mathrm{CC}$ events than for $\mathrm{NC}$ events, in which a hadron associated with the hadronic 
jet decays to a muon. Cuts based on this variable and on the reconstructed momentum compared to the reconstructed energy are an effective way to reduce all of the relevant beam related backgrounds. The distributions after the application of the preceding cuts are shown in figure 11, where the red lines illustrate the acceptance conditions defined in equations 9 and 10 ,

$$
\begin{array}{cccc}
E_{r e c} & \leq 5 \mathrm{GeV} \quad \text { or } & Q_{t}>0.25 \mathrm{GeV} / \mathrm{c} \text { and } \\
E_{r e c} \leq 7 \mathrm{GeV} \text { or } & & p_{\mu} \geq 0.3 \cdot E_{r e c} .
\end{array}
$$

QE like events (see section III B) and those events passing the conditions of equation 9 must also pass the conditions of equation 10 to remain in the data-set for the next series of cuts. The effect of these cuts is to reduce the background by a further order of magnitude, while only having a modest effect on the signal efficiency, as can be seen in table II.

\section{Cut summary}

In summary, after tuning the cuts described in the previous sub-sections to a test statistic, these were applied to independent simulated data leading to an absolute efficiency of $51 \%$ for $\nu_{\mu}$ selection and $62 \%$ for $\bar{\nu}_{\mu}$ selection, while reducing the background to a level below $10^{-3}$. A summary of all the cuts, with their effect on the signal and absolute background, can be found in Table II. The species which would be expected to contribute the greatest amount of background interactions for an example oscillation parameter set is also identified at each level.

\section{MIND RESPONSE TO THE GOLDEN CHANNEL}

Using a data-set of $3 \times 10^{6}$ events each of $\nu_{\mu} \mathrm{CC}, \bar{\nu}_{\mu} \mathrm{CC}, \nu_{e} \mathrm{CC}, \bar{\nu}_{e} \mathrm{CC}$ and $7 \times 10^{6} \mathrm{NC}$ interactions from neutrinos and anti-neutrinos generated using GENIE and tracked through the GEANT4 representation of MIND, the expected efficiency and background suppression for the reconstruction and analysis of the golden channel in MIND have been evaluated for both $\nu_{\mu}$ and $\bar{\nu}_{\mu}$ appearance. Additionally, the expected level of contamination of the signal from other appearance oscillation channels is considered. 

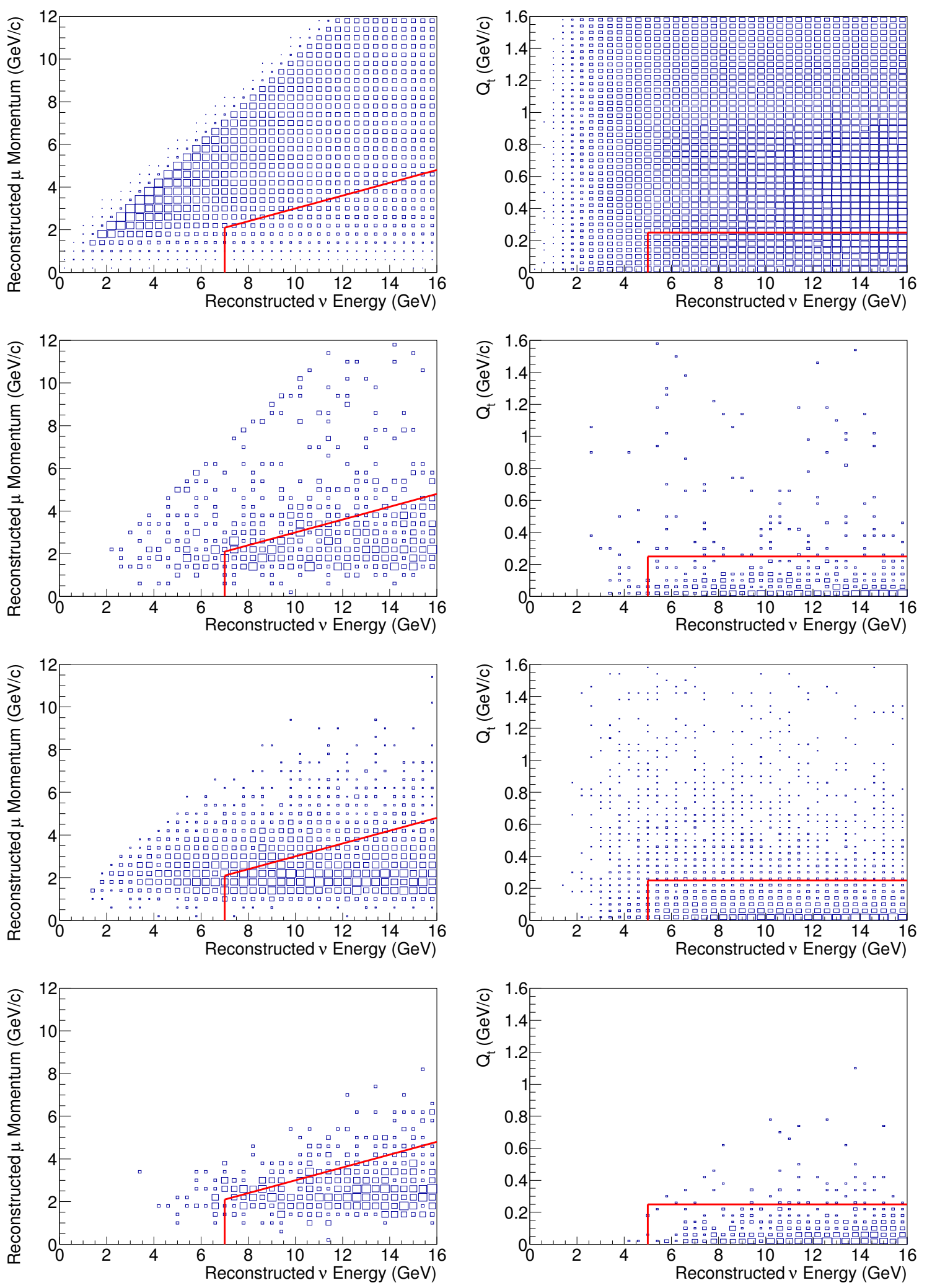

FIG. 11: Distributions of kinematic variables: (left) Reconstructed muon momentum with reconstructed neutrino energy for (top $\rightarrow$ bottom) $\nu_{\mu}\left(\bar{\nu}_{\mu}\right)$ signal, $\nu_{\mu}\left(\bar{\nu}_{\mu}\right)$ CC background, NC background, $\nu_{e}\left(\bar{\nu}_{e}\right)$ CC background and (right) $Q_{t}$ variable (in the same order). The red lines represent the cuts from equations 9 and 10 . 
TABLE II: Summary of cuts applied to select the golden channel appearance signals. The level of absolute efficiency and, for a 100 ktonne MIND $2000 \mathrm{~km}$ from the NF and $\theta_{13}=9.0^{\circ}$ and $\delta_{C P}=45^{\circ}$, the proportion of the total non-golden channel interactions remaining in the sample after each cut are also shown, along with the species contributing the greatest number of interactions.

\begin{tabular}{|c|c|c|c|c|c|}
\hline \multirow{2}{*}{ Cut } & Acceptance level & \multicolumn{2}{c|}{ Eff. after cut } & \multicolumn{2}{c|}{ background $\left(\times 10^{-3}\right)$} \\
\cline { 3 - 6 } & & $\nu_{\mu}$ & $\bar{\nu}_{\mu}$ & $\nu_{\mu}$ & $\bar{\nu}_{\mu}$ \\
\hline \hline & successful pattern rec. and fit & 0.91 & 0.93 & $419\left(\nu_{e}\right)$ & $153\left(\bar{\nu}_{\mu} N C\right)$ \\
\hline Fiducial & $z 1-z_{\text {end }} \leq 2000 \mathrm{~mm}$ & 0.88 & 0.90 & $400\left(\nu_{e}\right)$ & $147\left(\bar{\nu}_{\mu} N C\right)$ \\
\hline Max. momentum & $P_{\mu} \leq 16 \mathrm{GeV}$ & 0.85 & 0.89 & $158\left(\nu_{e}\right)$ & $108\left(\bar{\nu}_{e}\right)$ \\
\hline Fitted proportion & $N_{f i t} / N_{h} \geq 0.6$ & 0.81 & 0.87 & $74.4\left(\nu_{e}\right)$ & $71.3\left(\bar{\nu}_{e}\right)$ \\
\hline Track quality & $\mathcal{L}_{q / p}>-0.5$ & 0.70 & 0.76 & $13.6\left(\nu_{e}\right)$ & $20.3\left(\nu_{\tau}\right)$ \\
\hline Displacement & $d i s p X / d i s p Z>0.18-0.0026 N_{h}$ & 0.65 & 0.72 & $13.6\left(\nu_{\mu} \mathrm{NC}\right)$ & $10.9\left(\nu_{\tau}\right)$ \\
& $d i s p Z>6000 m m$ or $P_{\mu} \leq 3 d i s p Z$ & & & \\
\hline Quadratic fit & $q p_{\text {par }}<-1.0$ or $q p_{\text {par }}>0.0$ & 0.65 & 0.72 & $10.3\left(\nu_{\mu} \mathrm{NC}\right)$ & $10.9\left(\nu_{\tau}\right)$ \\
\hline CC selection & $\mathcal{L}_{1}>1.0$ & 0.63 & 0.70 & $2.1\left(\nu_{\mu} \mathrm{NC}\right)$ & $3.0\left(\nu_{\tau}\right)$ \\
\hline Kinematic & $E_{\text {rec }} \leq 5 \mathrm{GeV}$ or $Q_{t}>0.25$ & 0.51 & 0.62 & $0.3\left(\bar{\nu}_{\mu} \mathrm{NC}\right)$ & $0.9\left(\nu_{\tau}\right)$ \\
\hline & $E_{\text {rec }} \leq 7 \mathrm{GeV}$ or $P_{\mu} \geq 0.3 E_{\text {rec }}$ & & & & \\
\hline
\end{tabular}

\section{A. Signal efficiency and beam neutrino background suppression}

The resultant efficiencies for both polarities and the corresponding background levels expected for the appearance channels are summarised in Figs. 12 - 15. Numeric response matrices for each of the channels may be found in the Appendix. As can be seen in figure 12 the expected level of background from CC mis-identification is around $10^{-4}$, which is significantly below $10^{-3}$ at all energies for the new simulation and re-optimised analysis. This is also below the background levels achieved in [15], mainly due to the additional quality cuts.

The background from neutral current interactions lies at or below the $10^{-3}$ level, with the high energy region exhibiting a higher level than the low-energy region due to the dominance 

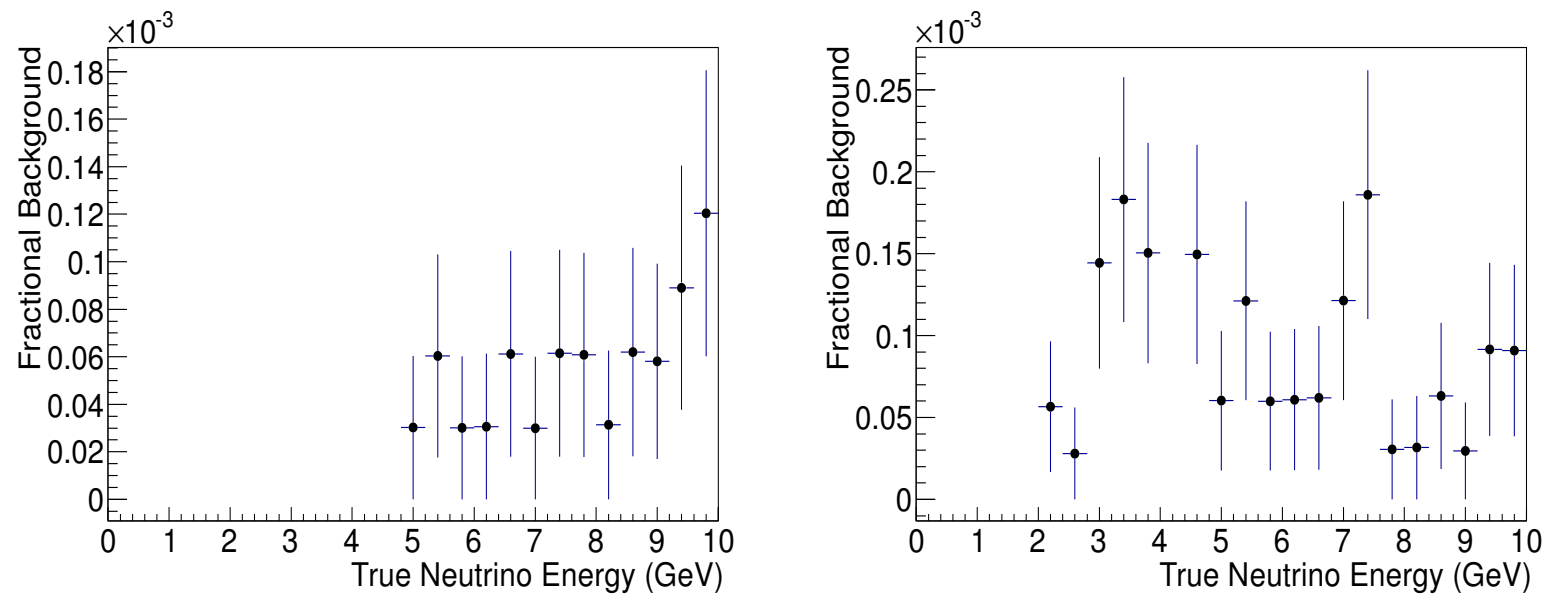

FIG. 12: Background from mis-identification of $\nu_{\mu}\left(\bar{\nu}_{\mu}\right) \mathrm{CC}$ interactions as the opposite polarity. (left) $\bar{\nu}_{\mu} \mathrm{CC}$ reconstructed as $\nu_{\mu} \mathrm{CC}$, (right) $\nu_{\mu} \mathrm{CC}$ reconstructed as $\bar{\nu}_{\mu} \mathrm{CC}$ as a function of true energy.
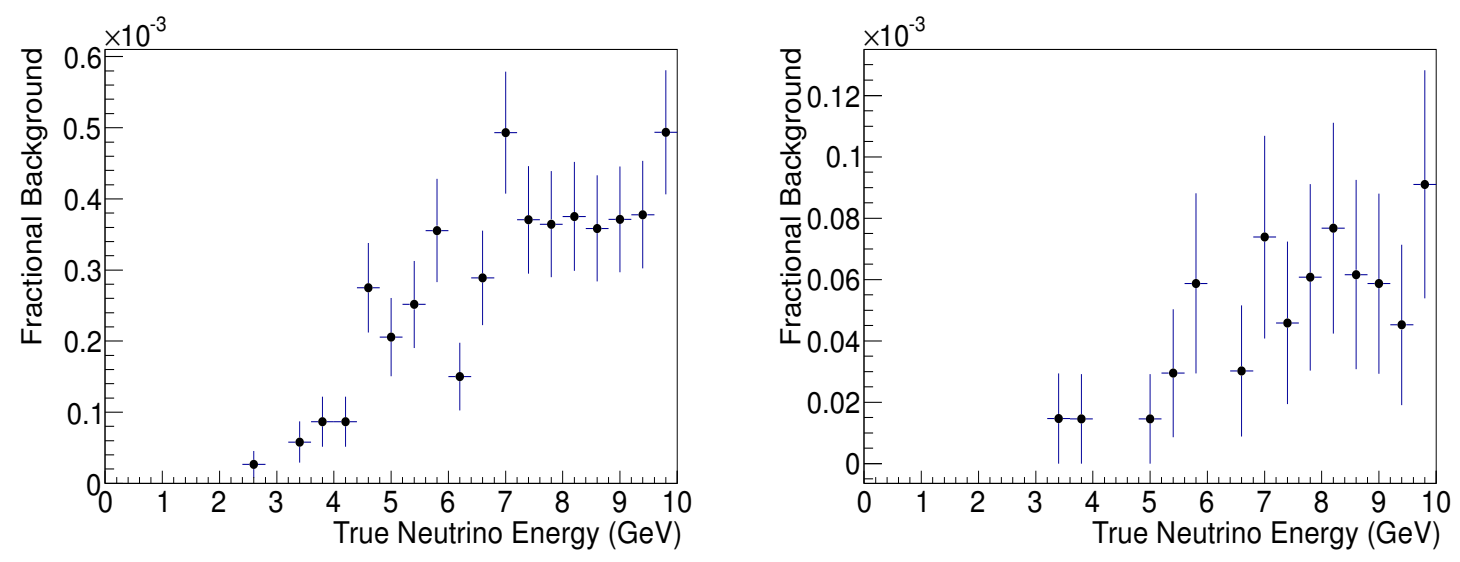

FIG. 13: Background from mis-identification of NC interactions as $\nu_{\mu}\left(\bar{\nu}_{\mu}\right) \mathrm{CC}$ interactions. (left) $\mathrm{NC}$ reconstructed as $\nu_{\mu} \mathrm{CC}$, (right) $\mathrm{NC}$ reconstructed as $\bar{\nu}_{\mu} \mathrm{CC}$ as a function of true energy.

of DIS interactions. The increased particle multiplicity and greater likelihood of producing a penetrating pion that can mimic a primary muon are the primary reasons for this increase. As expected, the NC background tends to be reconstructed at low energy due to the missing energy (see appendix $\mathrm{X}$ ).

The background from $\nu_{e}\left(\bar{\nu}_{e}\right)$ CC interactions is once again expected to constitute a very low level addition to the observed signal. This background is particularly well suppressed due 

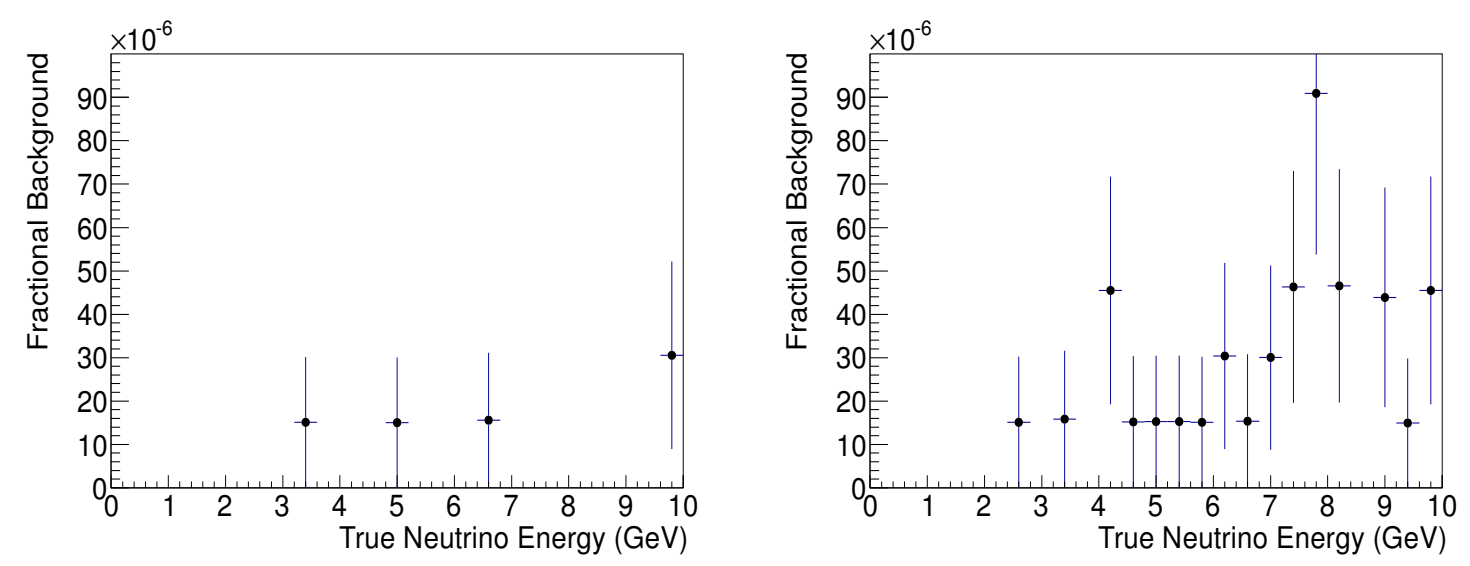

FIG. 14: Background from mis-identification of $\nu_{e}\left(\bar{\nu}_{e}\right) \mathrm{CC}$ interactions as $\nu_{\mu}\left(\bar{\nu}_{\mu}\right) \mathrm{CC}$ interactions. (left) $\nu_{e} \mathrm{CC}$ reconstructed as $\nu_{\mu} \mathrm{CC}$, (right) $\bar{\nu}_{e} \mathrm{CC}$ reconstructed as $\bar{\nu}_{\mu} \mathrm{CC}$ as a function of true energy.
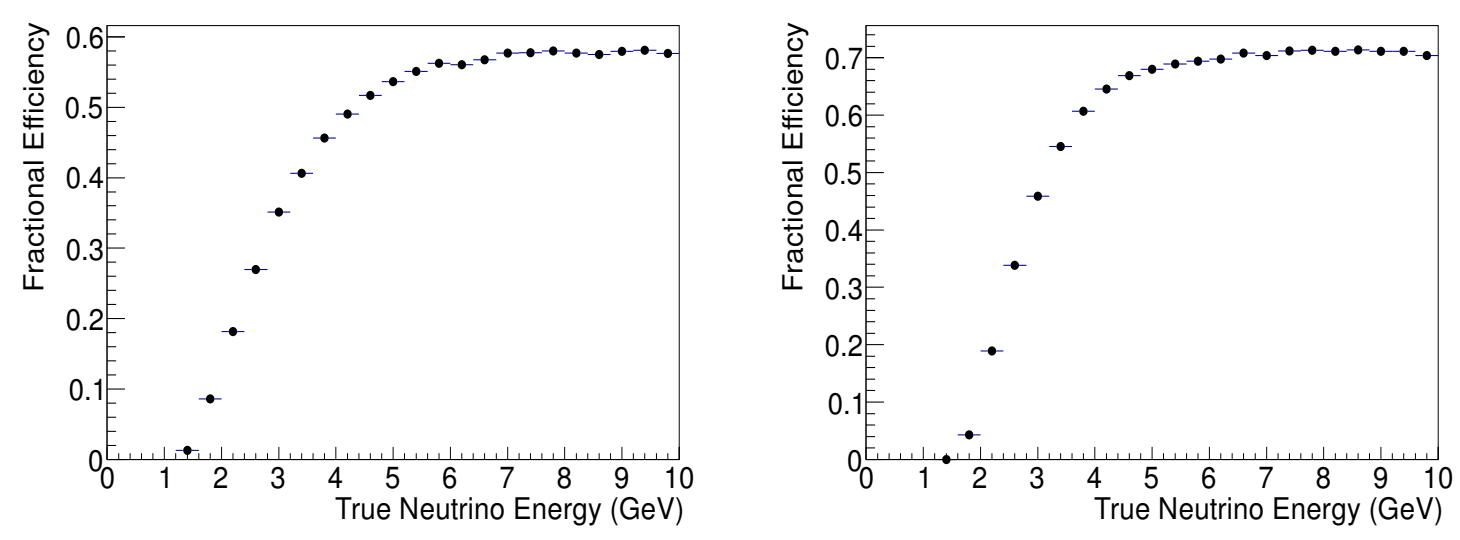

FIG. 15: Efficiency of reconstruction of $\nu_{\mu}\left(\bar{\nu}_{\mu}\right)$ CC interactions. (left) $\nu_{\mu}$ CC efficiency, (right) $\bar{\nu}_{\mu}$ CC efficiency as a function of true energy.

to the electron shower overlapping with the hadron shower. If a particle from the hadronic jet decays into a wrong-sign muon, it has a lower energy and is less isolated than in the NC case, so the kinematic cuts suppress this background more.

The efficiency of detection of the two $\nu_{\mu}$ polarities has a threshold lower than that seen in previous studies due to the presence of non-DIS interactions in the Monte Carlo sample. The efficiencies expected for the current analysis are shown in figure 15.

A comparison of the resultant $\nu_{\mu}$ and $\bar{\nu}_{\mu}$ efficiency can be made with that extracted in 
previous studies. Analyses performed in 2000 [4] and 2005 [38] assumed a $50 \mathrm{GeV}$ Neutrino Factory, so were optimised for high energy and low values of $\theta_{13}$. The background rejection achieved was at the level of $10^{-6}$, but at the expense of signal efficiency, especially below $10 \mathrm{GeV}$. A more recent analysis [9] was the first attempt at re-optimzing for a $25 \mathrm{GeV}$ Neutrino Factory, while that in 2010 [15] was still based on a GEANT3 model, but included full event reconstruction and a likelihood based analysis for the first time. There exists an improvement in threshold for the current analysis, between $2-3 \mathrm{GeV}$, due to the inclusion of $\mathrm{QE}$ and resonance events, since these events are easier to reconstruct (see figure 20.).

The difference in efficiency between the two polarities is effectively described by the difference in the inelasticity of neutrino and anti-neutrino CC interactions. Neutrino DIS interactions with quarks have a flat distribution in the Bjorken variable:

$$
y=\frac{E_{\nu}-E_{l}}{E_{\nu}}
$$

with $E_{l}$ the scattered-lepton energy. However, anti-neutrinos interacting with quarks follow $\mathrm{a} \propto(1-y)^{2}$ distribution [39. For this reason, neutrino interactions generally involve a greater energy transfer to the target. The efficiencies for the two species as a function of $y$ can be seen from figure 16-(left). The shape of the efficiency curves is a consequence of the ratio of DIS to non-DIS events in the event samples. Neutrino and anti-neutrino efficiencies are very similar, showing that the Bjorken $y$ of each event is the dominant contributor to the efficiency. The difference in neutrino and anti-neutrino efficiencies, when translated into true neutrino-energy, can be explained by the greater abundance of neutrino events at high $y$. However, since the cross section for the interaction of neutrinos is approximately twice that for anti-neutrinos, it is not expected that this reduced efficiency will affect the fit to the observed spectrum significantly.

\section{B. Contamination from oscillation channels containing $\nu_{\tau}$ or $\bar{\nu}_{\tau}$}

Three million events of both $\nu_{\tau}$ and $\bar{\nu}_{\tau}$ interactions were generated using the GENIE framework [16] and passed through the GEANT4 simulation of MIND. These events were then subject to the same digitization, reconstruction and analysis as the main beam backgrounds. Matrices were extracted describing the expected level of contamination in the golden channel data-set for the situation when a viable muon candidate from a $\nu_{\tau}\left(\bar{\nu}_{\tau}\right)$ interaction is reconstructed as a $\nu_{\mu}\left(\bar{\nu}_{\mu}\right)$ candidate with the same and opposite charge to the 

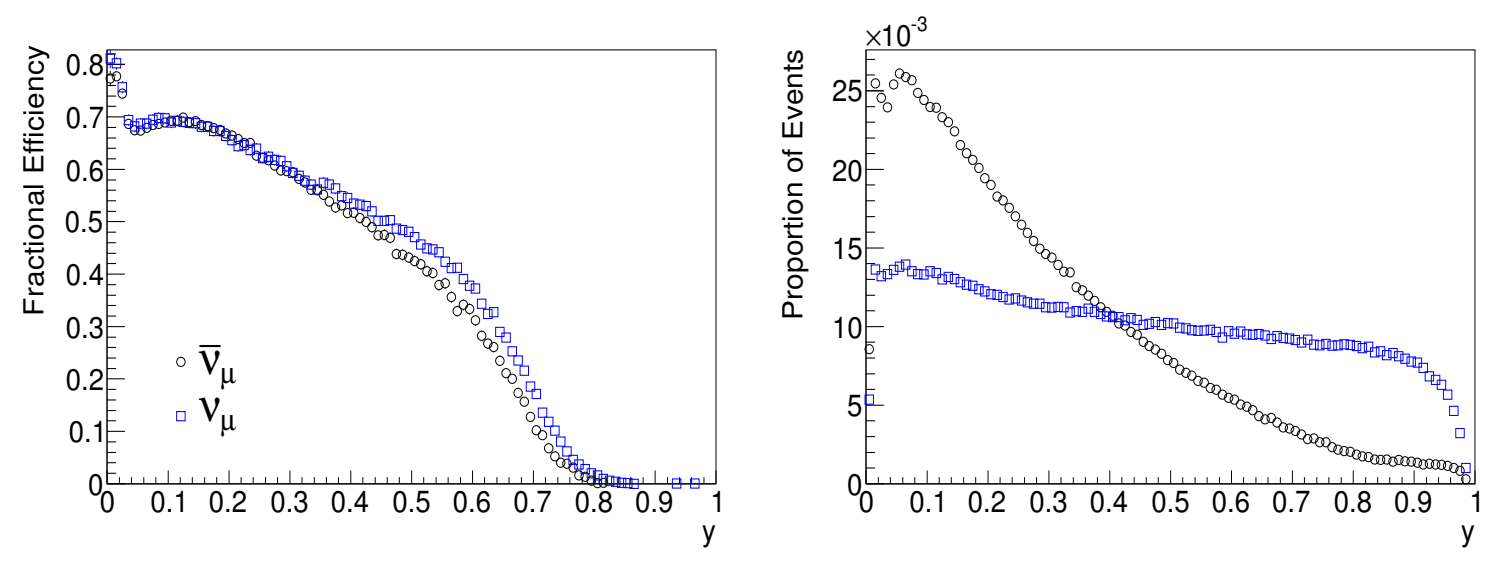

FIG. 16: $\nu_{\mu} \mathrm{CC}$ and $\bar{\nu}_{\mu} \mathrm{CC}$ signal detection efficiency as a function of $y$ (left) and the normalised distribution of all events considered in each polarity as a function of $y$ (right).

true primary $\tau$. As can be seen in figure 17 , between $1 \%$ and $3 \%$ of the $\nu_{\tau}\left(\bar{\nu}_{\tau}\right)$ interactions
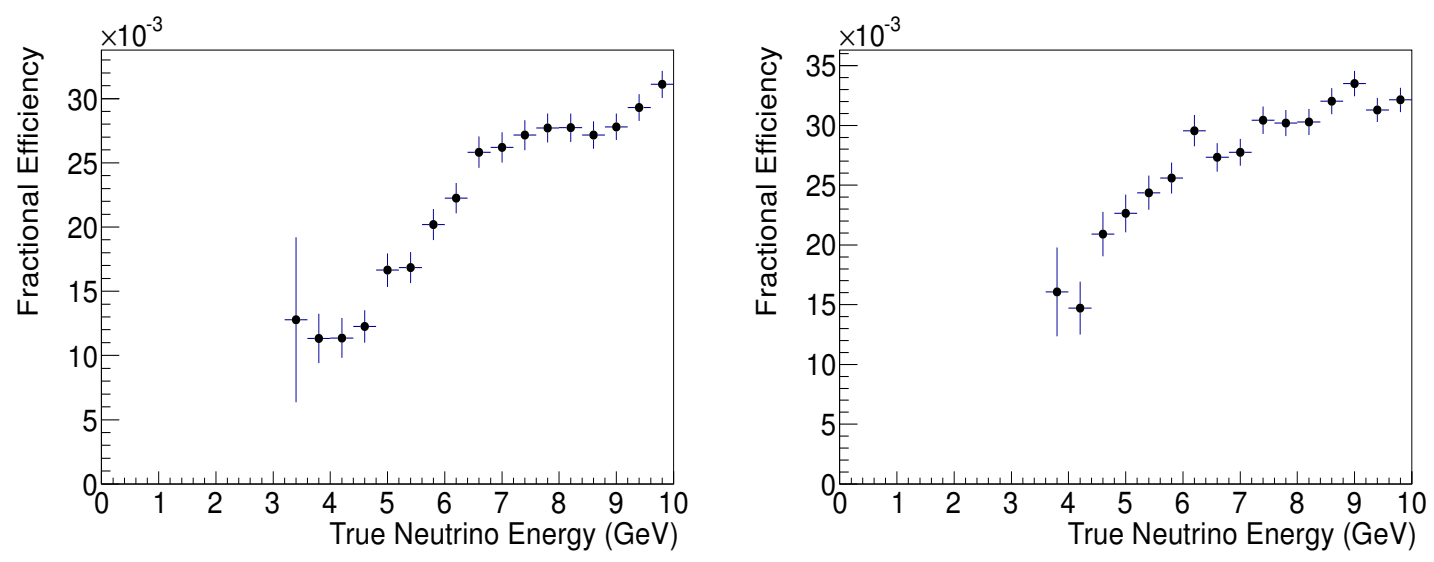

FIG. 17: Expected level of contamination from $\nu_{\tau}\left(\bar{\nu}_{\tau}\right)$ CC interactions due to the platinum channel. (left) $\nu_{\tau} \mathrm{CC}$ reconstructed as $\nu_{\mu} \mathrm{CC}$, (right) $\bar{\nu}_{\tau} \mathrm{CC}$ reconstructed as $\bar{\nu}_{\mu}$ $\mathrm{CC}$ as a function of true energy.

are expected to be identified as the golden $\nu_{\mu}\left(\bar{\nu}_{\mu}\right)$ interactions. Considered properly, this contamination should not weaken the extraction of the oscillation parameters (see [27]). Contamination from the dominant oscillation (which requires reconstruction with the opposite primary lepton charge) is expected to be below the $10^{-3}$ level (as shown in figure 18). This contamination is taken into account, but does not deteriorate the $\delta_{C P}$ fits, since the dominant oscillation is less sensitive to this parameter for large values of $\theta_{13}$. 

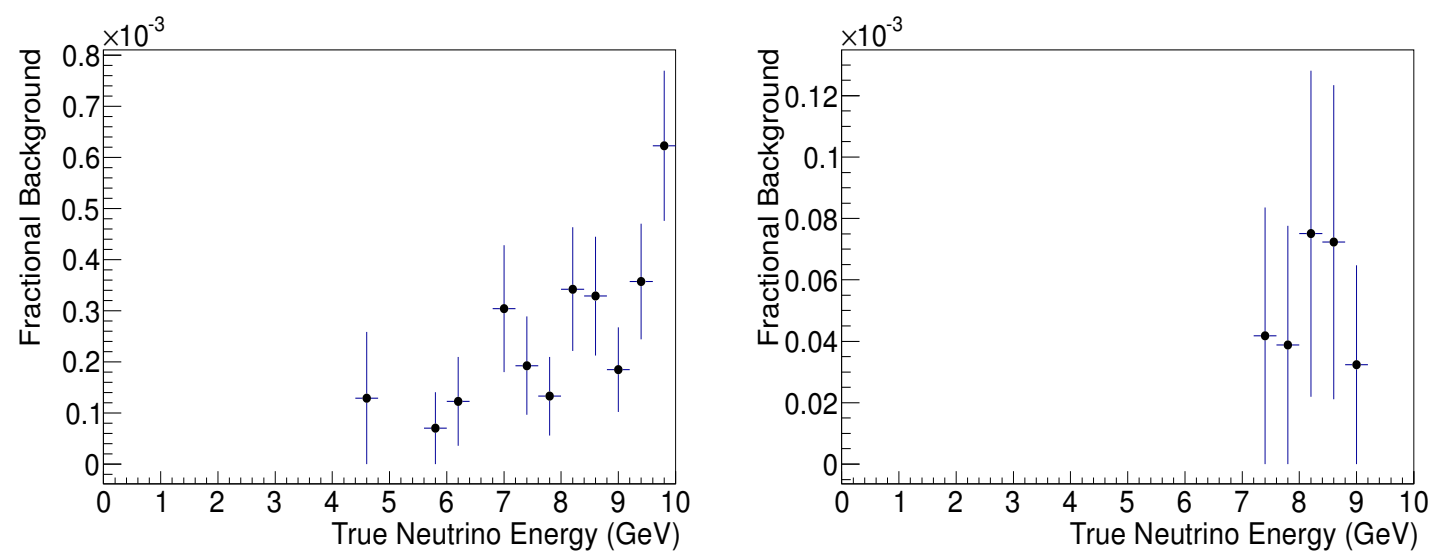

FIG. 18: Expected level of contamination from $\nu_{\tau}\left(\bar{\nu}_{\tau}\right)$ CC interactions due to the dominant oscillation. (left) $\bar{\nu}_{\tau} \mathrm{CC}$ reconstructed as $\nu_{\mu} \mathrm{CC}$, (right) $\nu_{\tau} \mathrm{CC}$ reconstructed as $\bar{\nu}_{\mu} \mathrm{CC}$ as a function of true energy.

\section{Interaction expectation for $10^{21}$ muon decays}

Using the response matrices extracted using the analysis described in the preceding sections it is possible to make a prediction of the expected contribution to the Monte Carlo sample from each of the neutrino types in the beam. Figure 19 shows the expected number of events for the best fit values of the currently measured parameters taken from [40]: $\theta_{12}=33.5^{\circ}, \theta_{23}=45^{\circ}, \Delta m_{21}^{2}=7.65 \times 10^{-5} \mathrm{eV}^{2}, \Delta m_{32}^{2}=2.4 \times 10^{-3} \mathrm{eV}^{2}$ for $\delta_{C P}=45^{\circ}$ and calculating matter effects using the PREM model [41]. The number of interactions were calculated for a 100 ktonne MIND at a distance of $2000 \mathrm{~km}$ from the NF for a value of $\theta_{13}=9.0^{\circ}$, for an integrated flux due to $10^{21}$ decays of each polarity in the straight sections of the decay pipes.

\section{STUDY OF SYSTEMATIC UNCERTAINTIES}

The efficiencies and backgrounds described above will be affected by several systematic effects. There will be many contributing factors including uncertainty in the determination of the parameters used to form the cuts in the analysis, uncertainty in the exclusive cross-sections, uncertainty in the determination of the hadronic shower energy and direction resolution, and any assumptions in the representation of the detector and readout. While 

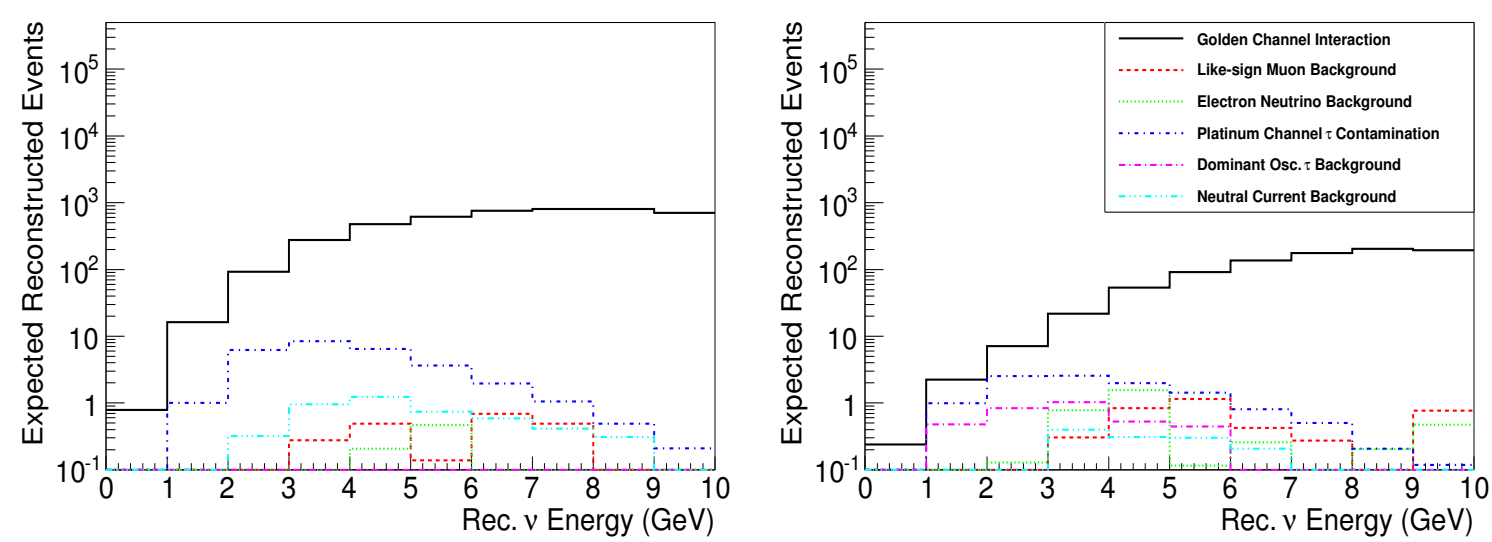

FIG. 19: Expected interactions in a 100 ktonne MIND $2000 \mathrm{~km}$ from the Neutrino Factory for $\theta_{13}=9.0^{\circ}$. Left column for $\nu_{\mu}$ appearance and right for $\bar{\nu}_{\mu}$ appearance.

exact determination of the overall systematic error in the efficiencies is complicated, an estimate of the contribution of different factors can be obtained by setting certain variables to the extremes of their errors.

The exclusive QE, DIS and 'other' cross-sections in the data sample could have a significant effect on the signal efficiencies and backgrounds. The efficiencies for the reconstruction of true QE and true DIS interactions are compared to the nominal efficiency in figure 20 where the dominance of DIS interactions in the backgrounds is clear. Although experimental data are available, confirming the presence of non-DIS interactions in the energy region of interest, there are significant errors in the transition regions (see for example [42, 43]). These errors lead to an uncertainty in the proportion of the different types of interaction that can affect the efficiencies. In order to study the systematic error associated with this effect, events of certain types were randomly removed from the data-set and the mean effect quantified. As an illustration of the method, consider the contribution from QE interactions. Taking the binned errors on the cross-section measurements from [42, 43], a run to reduce the QE content would exclude a proportion of events in a bin so that instead of contributing the proportion $\frac{N_{Q E}}{N_{t o t}}$, where $N_{Q E}$ and $N_{t o t}$ are the number of QE interactions and the total number of interactions in the bin of interest, it would instead contribute: $\frac{N_{Q E}-\sigma_{Q E} N_{Q E}}{N_{t o t}-\sigma_{Q E} N_{Q E}}$, where $\sigma_{Q E}$ is the proportional error on the QE cross section for the bin. Since the data-set is finite and an actual increase in the number of QE interactions is not possible, the equivalent run to increase the QE contribution reduces the contribution of the "rest" by an amount 

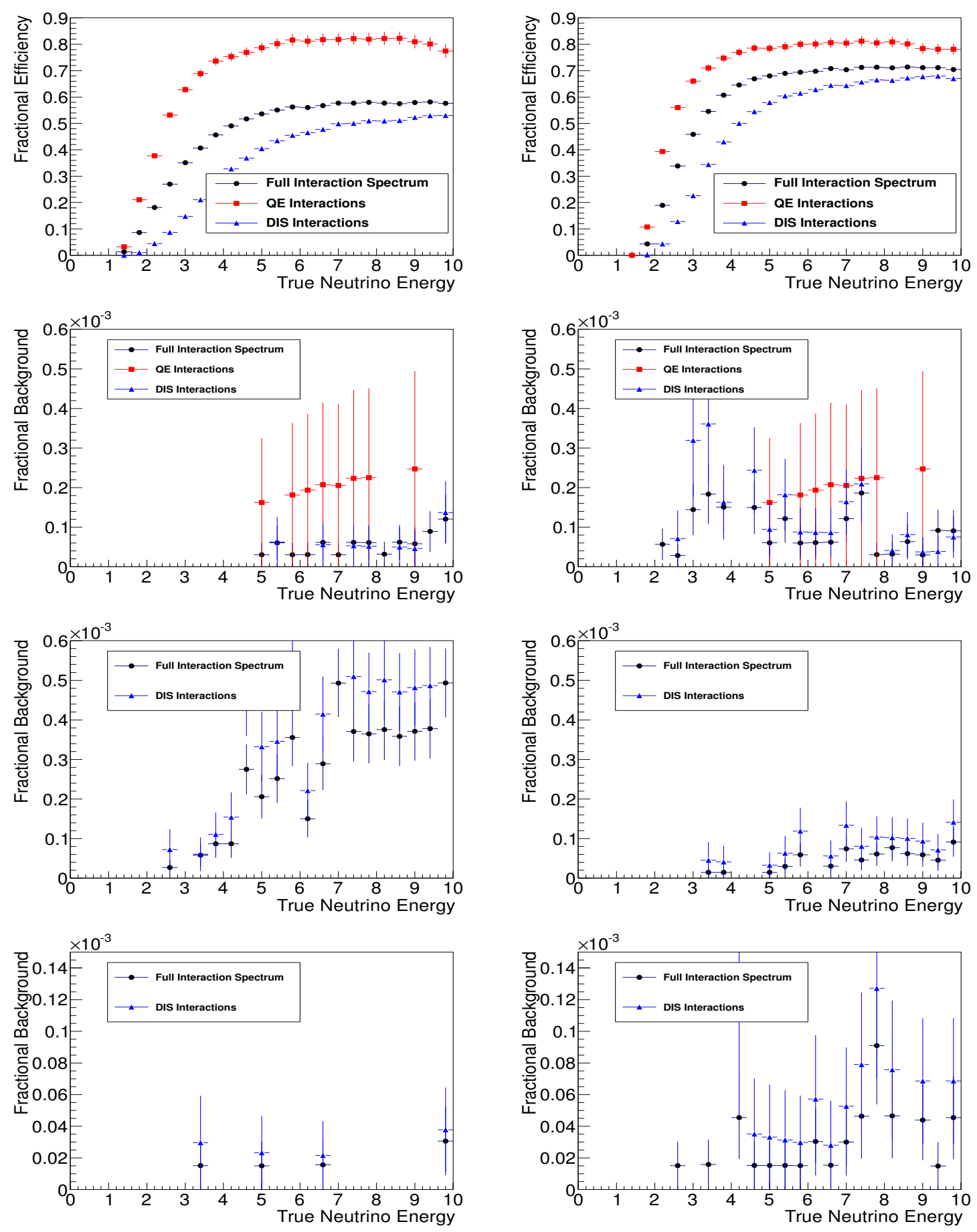

FIG. 20: Efficiencies for a pure DIS sample compared to the nominal case. (top) Signal efficiency, (second line) $\nu_{\mu}\left(\bar{\nu}_{\mu}\right)$ CC background, (third line) NC background and (bottom) $\nu_{e}\left(\bar{\nu}_{e}\right)$ CC background. $\nu_{\mu}$ appearance on the left and $\bar{\nu}_{\mu}$ appearance on the right. 
calculated to give the corresponding proportional increase in QE interactions:

$$
\frac{N_{Q E}+\sigma_{Q E} N_{Q E}}{N_{t o t}+\sigma_{Q E} N_{Q E}}=\frac{N_{Q E}}{N_{t o t}-\epsilon N_{\text {rest }}} ;
$$

where $N_{\text {rest }}$ is the total number of non-QE interactions in the bin and $\epsilon$ is the required proportional reduction in the 'rest' to simulate an appropriate increase in QE. Solving for $\epsilon$ yields the required reduction:

$$
\epsilon=\frac{\sigma_{Q E}}{1+\sigma_{Q E}}
$$

The $1 \sigma$ systematic error can be estimated as the mean difference between the nominal efficiency and the increase due to a higher QE proportion or decrease due to exclusion. The errors in the true $\nu_{\mu}$ and $\bar{\nu}_{\mu}$ efficiencies extracted using this method that varies the contribution of QE, $1 \pi$ and other non-DIS interactions are shown in figure 21. Errors for $1 \pi$ resonant reactions are estimated to be $\sim 20 \%$ below $5 \mathrm{GeV}$ (as measured by the $\mathrm{K} 2 \mathrm{~K}$ near detector [44]) and at 30\% above. Due to the large uncertainty, both theoretically and experimentally, on the models describing other resonances, coherent, diffractive and elastic processes, a very conservative error of $50 \%$ is taken when varying the contribution of the "others". As can be seen in figure 21, the systematic effect is less than $1 \%$ for neutrino energies between $3 \mathrm{GeV}$ and $10 \mathrm{GeV}$ increasing to $4 \%$ near $1 \mathrm{GeV}$, with increased QE and $1 \pi$ interactions generally increasing the efficiency and increased contribution of the "other" interactions having the effect of decreasing efficiency. This last result is likely to be predominantly due to resonances producing multiple tracks. The effect on backgrounds is expected to be minimal, as was also shown in figure 20. At the time of a Neutrino Factory, the cross-section uncertainties should be much smaller than the ones assumed here, so we expect the systematic error to be below $1 \%$ for all energies.

Another important source of systematic uncertainty is due to the error in the muon momentum and the hadron shower energy used to reconstruct the total neutrino energy. The muon is fully reconstructed, but the hadron shower reconstruction is performed assuming a parametrisation to the hadron energy and angular resolution. The particular choice of this parametrisation introduces a systematic error on the resulting signal and background efficiencies that should be studied. Taking a $6 \%$ error as quoted for the energy scale uncertainty assumed by the MINOS collaboration [7] and varying the constants of the energy and direction smears by this amount, it can be seen (blue bands in figure 22 that, to this level, 

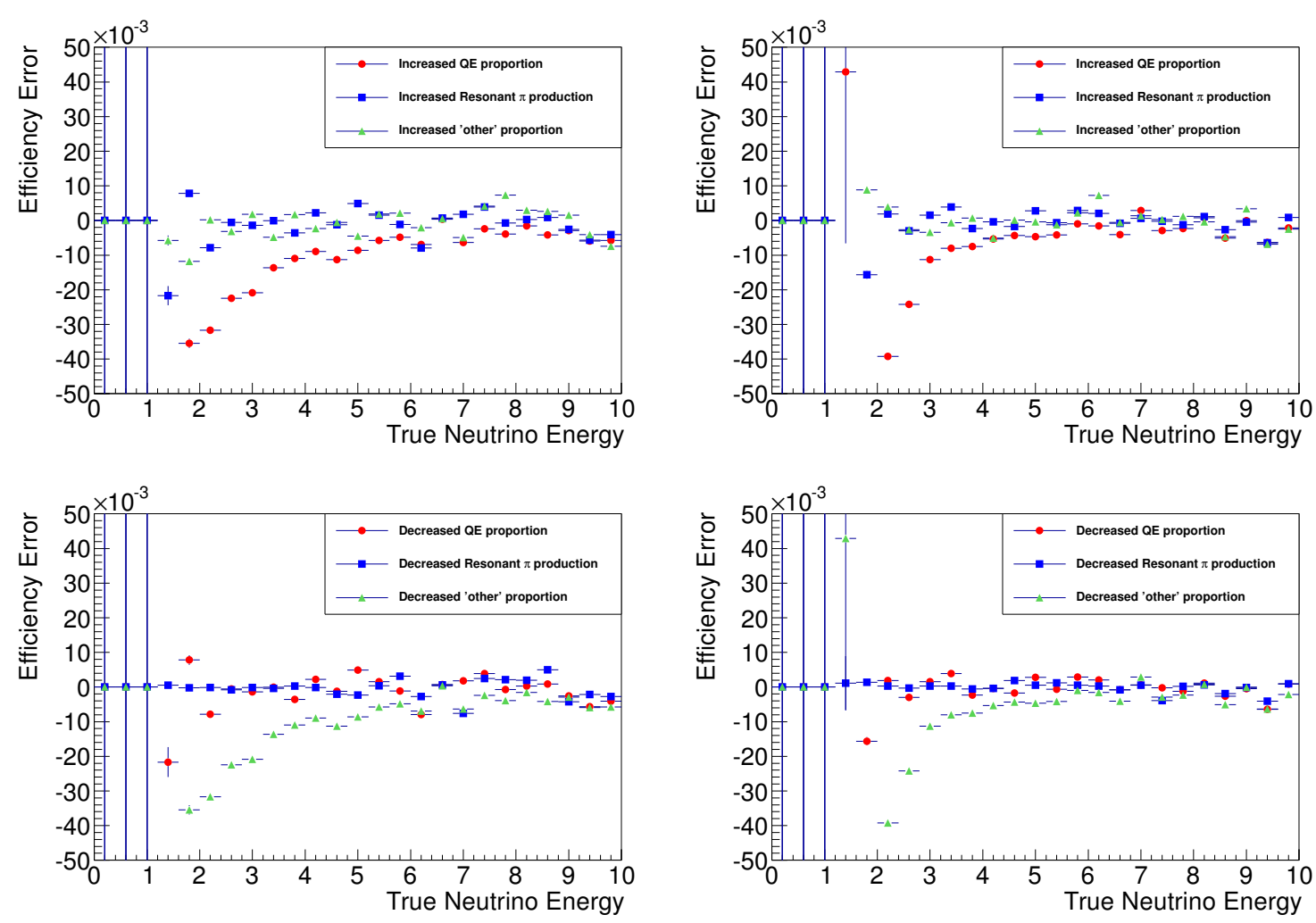

FIG. 21: Calculated error on signal efficiencies on increasing (top) and decreasing (bottom) the proportion of non-DIS interactions in the data-set. (left) Errors on true energy $\nu_{\mu} \mathrm{CC}$ efficiency and (right) errors on true energy $\bar{\nu}_{\mu} \mathrm{CC}$ efficiency

the hadronic resolutions have little effect on the true neutrino-energy efficiencies. However, the hadronic direction resolution is likely to have far greater uncertainty and would be very sensitive to noise in the readout electronics. Also shown in figure 22 are the efficiencies when the hadronic energy resolution parameters are $6 \%$ larger but with a $50 \%$ increase in the angular resolution parameters. We expect in a real detector to measure the hadronic angular resolution with a precision of better than $50 \%$, even though we cannot quantify this precision yet. However, even at this level, the observed difference in efficiency is only at the level of $1 \%$ above $7-8 \mathrm{GeV}$. A combination of the exclusive cross-sections and hadronic energy uncertainties implies a total systematic uncertainty for the measurement efficiency of order $1 \%$ over the neutrino energy range above $2 \mathrm{GeV}$. 

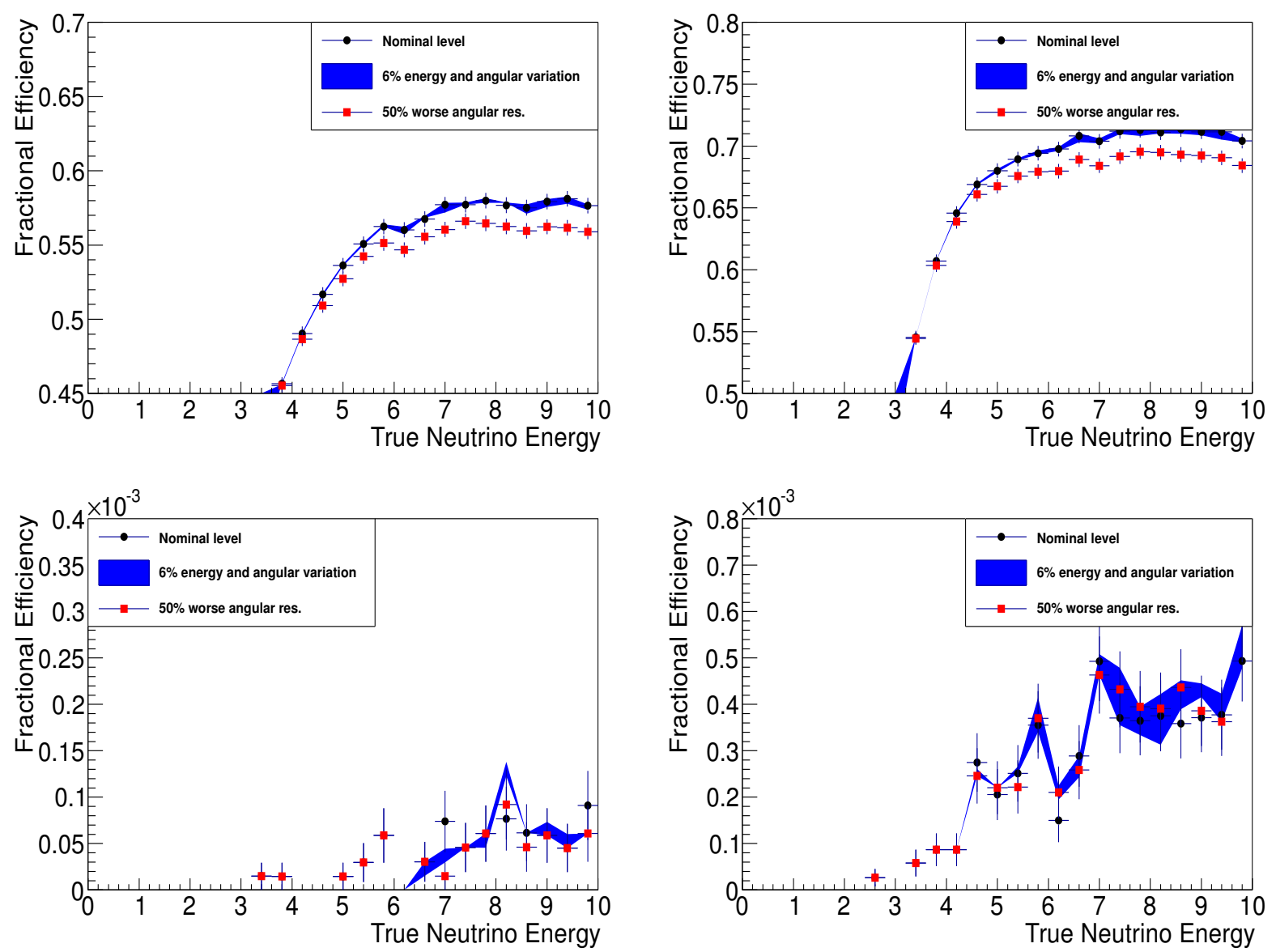

FIG. 22: Variation of signal efficiency (top) and NC backgrounds (bottom) due to a 6\% variation in the hadron shower energy and direction resolution and a more pessimistic 50\% reduction in angular resolution (focused on region of greatest variation).

\section{COMPARISON BETWEEN EVENT GENERATORS}

The above analysis uses the GENIE event generator to simulate the interaction of events with matter in the detector. This generator was assumed to bring this effort in line with current experiments, such as MINOS, where good agreement with data has been achieved. It is useful to compare to a previous version of this analysis with the NUANCE event generator [20]. A comparison of the neutrino charge current detection efficiencies appears in figure 23. This shows that the GENIE derived analysis produces smaller positive identification of charge current events. This loss of performance is linear with respect to neutrino energy for energies greater than $5 \mathrm{GeV}$, so that the analysis of the GENIE simulation is $20 \%$ less efficient 


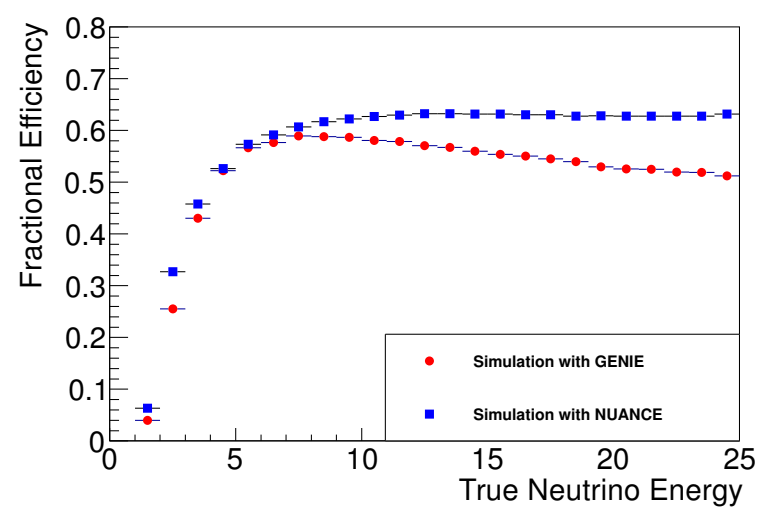

(a) GENIE and NUANCE $\nu_{\mu}$ CC efficiency

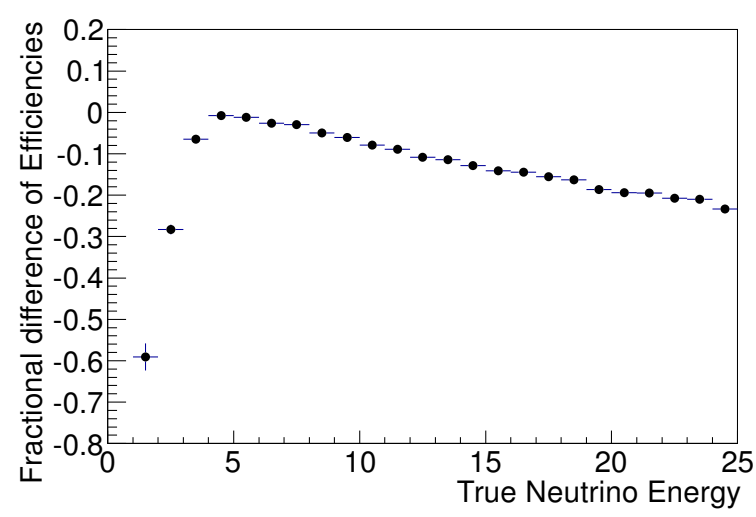

(c) Fractional difference between GENIE and

NUANCE $\nu_{\mu}$ CC efficiencies

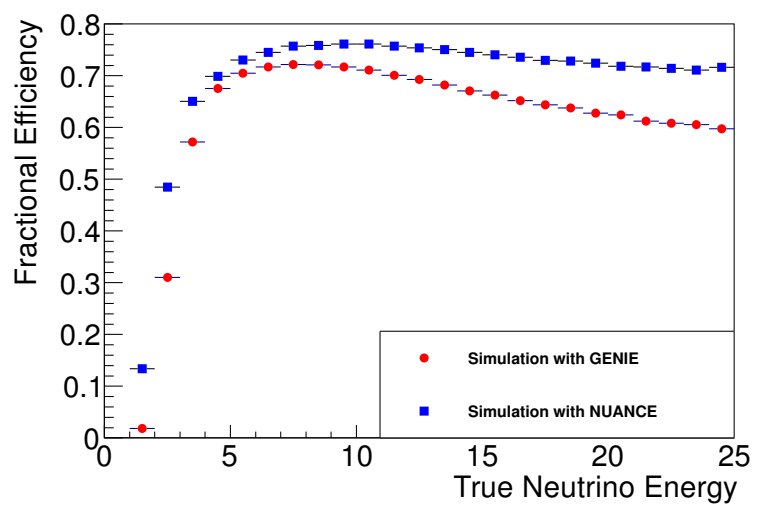

(b) GENIE and NUANCE $\bar{\nu}_{\mu}$ CC efficiency

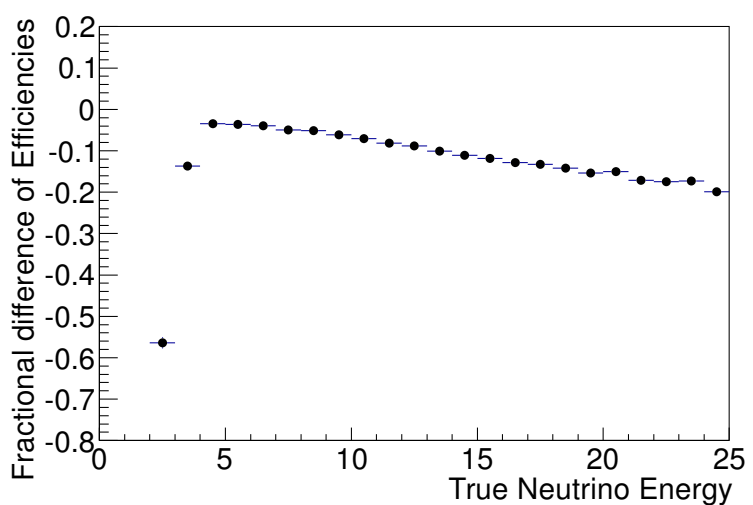

(d) Fractional difference between GENIE and

NUANCE $\bar{\nu}_{\mu} \mathrm{CC}$ efficiencies

FIG. 23: The Golden channel analysis efficiencies from simulations of charge current neutrino events in a MIND detector generated using GENIE and NUANCE neutrino generators. The differences between the efficiencies as fractions of the GENIE efficiency are also shown.

than that of the NUANCE simulation. This difference is partially ascribed to differences in the parton distribution functions used by each generator and should not be interpreted as a systematic error of the analysis, since the measured event rates in a future experiment will be cross-checked against the simulations, so the efficiencies will be determined much more accurately than the difference between generators. We assume that GENIE, which has been benchmarked against recent neutrino experiments, serves as a more realistic estimator for the efficiency of the analysis. 


\section{MIND SENSITIVITY}

The Neutrino Factory is required to measure the CP-violating phase $\delta_{C P}$ simulaneously with $\theta_{13}$ while removing ambiguity caused by degenerate solutions. Extracting the oscillation parameters from the observed signal at the far detectors requires the accurate prediction of the expected flux without oscillation, which is used along with the calculated oscillation probabilities to fit the observed signal for the best value of $\theta_{13}$ and $\delta_{C P}$. Due to the large distance to the far detectors, the flux spectra expected from the decay rings of the Neutrino Factory are accurately approximated by the flux from a point source of muons travelling with appropriate Lorentz boost in the direction of the detector. Using this flux or, alternatively, a projection of the spectra observed in the near detector, the number of true interactions expected in MIND as a function of energy can be calculated for each value of $\theta_{13}$ and $\delta$. These spectra can then be multiplied by the response matrices shown in the Appendix to calculate the observed golden channel interaction spectrum expected for some hypothetical values of the oscillation parameters.

A Neutrino Factory storing muons of energy $10 \mathrm{GeV}$ is assumed, of which $5.0 \times 10^{20}$ per year of each species decay in the straight sections pointing towards the MIND far detector of 100 ktonnes mass placed at a distance of $2000 \mathrm{~km}$ from the facility.

\section{A. The NuTS framework}

The Neutrino tool suite (NuTS) was developed for the studies presented in [45 47]. It provides a framework for the generation of appropriate fluxes for different neutrino accelerator facilities along with the necessary infrastructure to calculate the true neutrino oscillation probabilities for all channels. In addition, using the parametrisation of the total interaction spectra calculated in section IIIA, the expected number of events in a given energy bin can be calculated. Using this framework and the response matrices extracted for MIND, simulated data for an experiment can be generated as

$$
\operatorname{Data}_{\text {sim }}^{i, j}=\operatorname{smear}\left(M_{s i g}^{i} N_{s i g}^{i, j}+\sum_{k} M_{b k g}^{i, k} N_{b k g}^{i, j, k}\right),
$$

for each polarity and detector baseline of interest, where $M_{\text {sig }}^{i}$ is the response matrix for MIND for a particular signal channel $i$ (stored $\mu^{+}$or $\mu^{-}$), $N_{\text {sig }}^{i, j}$ is the $100 \%$ efficiency interaction spectrum in true $\nu$ energy bins for a channel $i$ at a detector baseline $j$ (in this case, 
there is only one baseline at $2000 \mathrm{~km}$ ), $M_{b k g}^{i, k}$ is the response matrix for a background $k$ (misidentification of CC interactions from other neutrino species or from NC) to the appearance channel $i, N_{b k g}^{i, j, k}$ is the $100 \%$ expectation spectrum for a background $k$ to an appearance signal $i$ at a detector baseline $j$ and these expected values are used to calculate an observed number of interactions following a Poisson distribution.

\section{B. Fitting for $\theta_{13}$ and $\delta_{C P}$ simultaneously}

Due to the correlation between $\theta_{13}$ and $\delta_{C P}$, a simultaneous fit is necessary. Defining a grid of $\theta_{13}$ and $\delta_{C P}$ values the $\chi^{2}$ of a fit to $D a t a_{s i m}^{i, j}$ can be calculated using the function

$$
\begin{gathered}
\chi^{2}=\sum_{j}\left\{2 \times \sum_{e}^{E_{\mu}}\left(A_{j} x_{j} N_{+, j}^{e}\left(\theta_{13}, \delta_{C P}\right)-n_{+, j}^{e}+n_{+, j}^{e} \log \left(\frac{n_{+, j}^{e}}{A_{j} x_{j} N_{+, j}^{e}\left(\theta_{\left.13, \delta_{C P}\right)}\right.}\right)\right.\right. \\
\left.+A_{j} N_{-, j}^{e}\left(\theta_{13}, \delta_{C P}\right)-n_{-, j}^{e}+n_{-, j}^{e} \log \left(\frac{n_{-, j}^{e}}{A_{j} N_{-, j}^{e}\left(\theta_{13}, \delta_{C P}\right)}\right)\right) \\
\left.+\frac{\left(A_{j}-1\right)^{2}}{\sigma_{A}}+\frac{\left(x_{j}-1\right)^{2}}{\sigma_{x}}\right\}
\end{gathered}
$$

where $n_{i, j}^{e}$ is the simulated data $\left(D a t a_{s i m}^{i, j}\right)$ for an energy bin $e, N_{i, j}^{e}\left(\theta_{13}, \delta_{C P}\right)$ is the predicted spectrum for the values of $\theta_{13}$ and $\delta_{C P}$ represented by the grid point (calculated as in equation 14 but without a smear) and $j$ represents the baseline as in equation 14 . The uncertainty in the expected number of interactions and expected ratio in interactions between neutrinos and antineutrinos are represented by the additional free parameters $A_{j}$ and $x_{j}$ respectively and their corresponding errors.

We made two assumptions regarding the overall event normalisation: one assumes a conservative error of $\sigma_{A}=0.025$ and the other assumes a more optimistic (but realistic) assumption for a neutrino factory of $\sigma_{A}=0.01$. The uncertainty in the ratio of cross sections between neutrinos and antineutrinos is maintained fixed at $\sigma_{x}=0.01$, which is the level to which a near detector would seek to measure the interaction cross-sections at the time of a neutrino factory. The minimisation of the parameters $A$ and $x$ is performed analytically for each predicted dataset to leading order. The contours at $\chi_{\min }^{2}+9$ represent approximately the $3 \sigma$ level of understanding and those at $\chi_{\min }^{2}+25$ represent $5 \sigma$. In such a fit, the experimentally determined oscillation parameters [40] are considered fixed. While there would be some systematic error associated with uncertainty in these parameters, systematics 


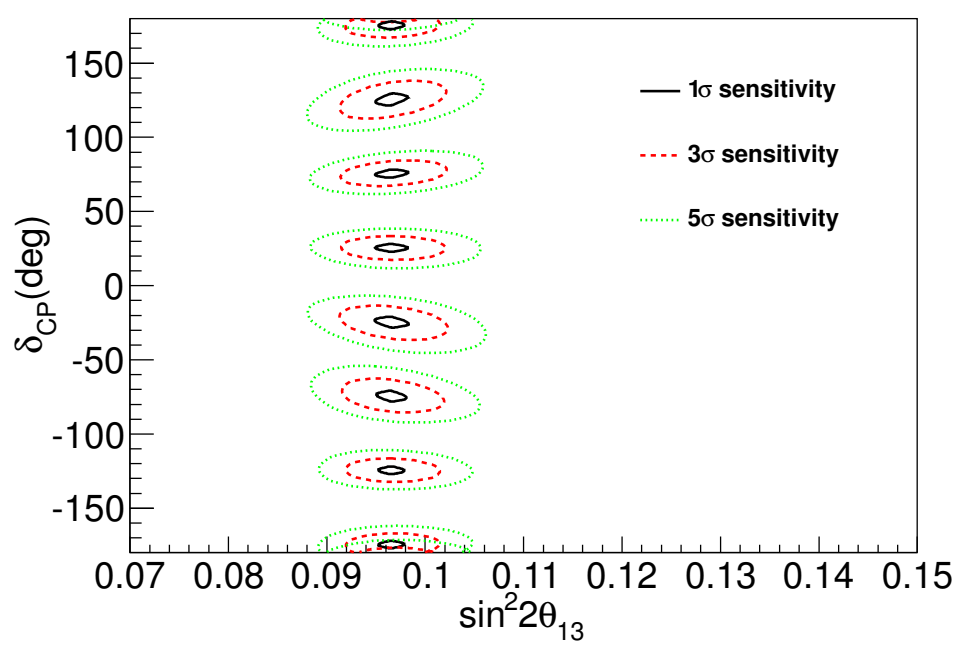

FIG. 24: Examples of $1 \sigma, 3 \sigma$ and $5 \sigma$ fits to simulated data for the $10 \mathrm{GeV}$ Neutrino Factory, for $\sin ^{2} 2 \theta_{13}=0.096$.

from the normalisation and cross-section uncertainties are expected to dominate. Some examples for such fits, assuming $\sin ^{2} 2 \theta_{13}=0.096$, are shown in figure 24 .

\section{Sensitivity to the mass hierarchy}

Distinguishing between the two possible mass hierarchies is important for the understanding of the neutrino mass sector. The sensitivity to the true mass hierarchy is defined here as when the true sign of $\Delta m_{13}^{2}$ can be distinguished from the opposite sign to the appropriate $n \sigma$ level, that is

$$
\chi_{\min }^{2}\left(-\Delta m_{13}^{2}\right)-\chi_{\min }^{2}\left(\Delta m_{13}^{2}\right) \geq n^{2}
$$

Sensitivity to the mass hierarchy up to $5 \sigma$ covers the whole region down to $\sin ^{2} 2 \theta_{13}<10^{-4}$, so the Neutrino Factory would be able to cover easily the current value of $\sin ^{2} 2 \theta_{13} \sim 0.1$.

\section{Sensitivity to $\delta_{C P}$}

Sensitivity to the measurement of $\delta_{C P}$ is defined as when the difference between the minimum $\chi^{2}$ with respect to the minimum obtained by fitting with $C P$ conserving cases is 

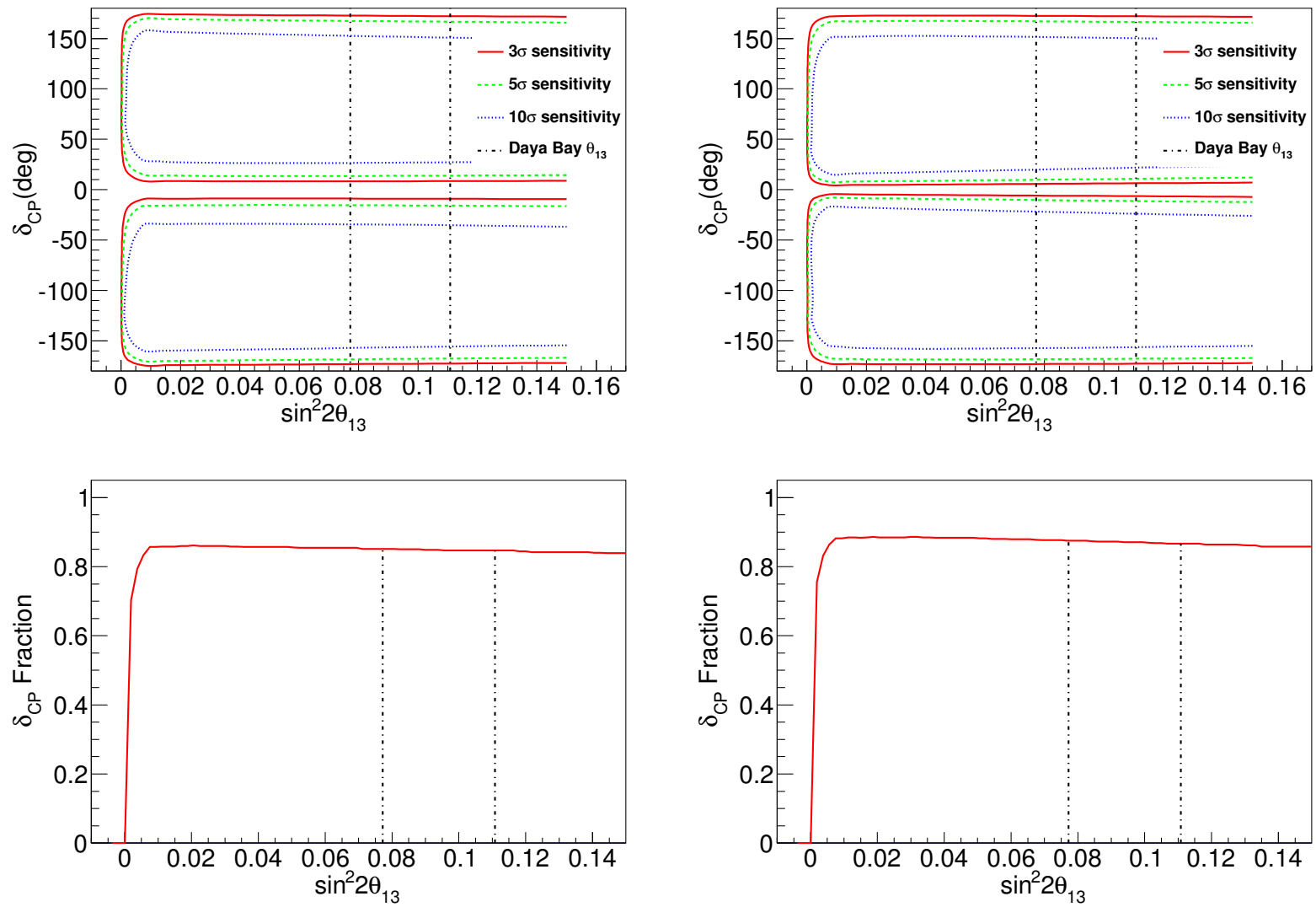

FIG. 25: $\delta_{C P} 3 \sigma, 5 \sigma$ and $10 \sigma$ measurements (top) and $\delta_{C P} 5 \sigma$ coverage to measure $C P$ violation (bottom) as a function of $\sin ^{2} 2 \theta_{13}$ for true normal hierarchy (left) and true inverted hierarchy (right). The vertical lines represent the range of possible values of

$$
\sin ^{2} 2 \theta_{13} \text {. }
$$

greater than the appropriate $n \sigma$ level, that is

$$
\min \left(\chi^{2}\left(\delta_{C P}=0\right), \chi^{2}\left(\delta_{C P}=180\right), \chi^{2}\left(\delta_{C P}=-180\right)\right)-\chi_{\min }^{2} \geq n^{2} .
$$

The $\delta_{C P}$ measurement and $\delta_{C P}$ coverage plots to measure $\mathrm{CP}$ violation by the $10 \mathrm{GeV}$ Neutrino Factory are shown in figure 25 for the normal mass hierarchy (left) and for the inverted mass hierarchy (right). The $\mathrm{CP}$ coverage to measure $\delta_{C P}$ at the $5 \sigma$ level for both normal and inverted mass hierarchy is $\sim 85 \%$ in the range of the currently measured values of $\sin ^{2} 2 \theta_{13}$. These results improve those presented by the Interim Design Report [20].

The accuracy achieved in the measurement of $\delta_{C P}$ is an increasingly important parameter to determine the performance of a facility [48]. The $1 \sigma$ error $\Delta \delta_{C P}$ for $\theta_{13}=9^{\circ}$ is shown in figure 26 (left), under two assumptions of the overall normalisation and cross-section 

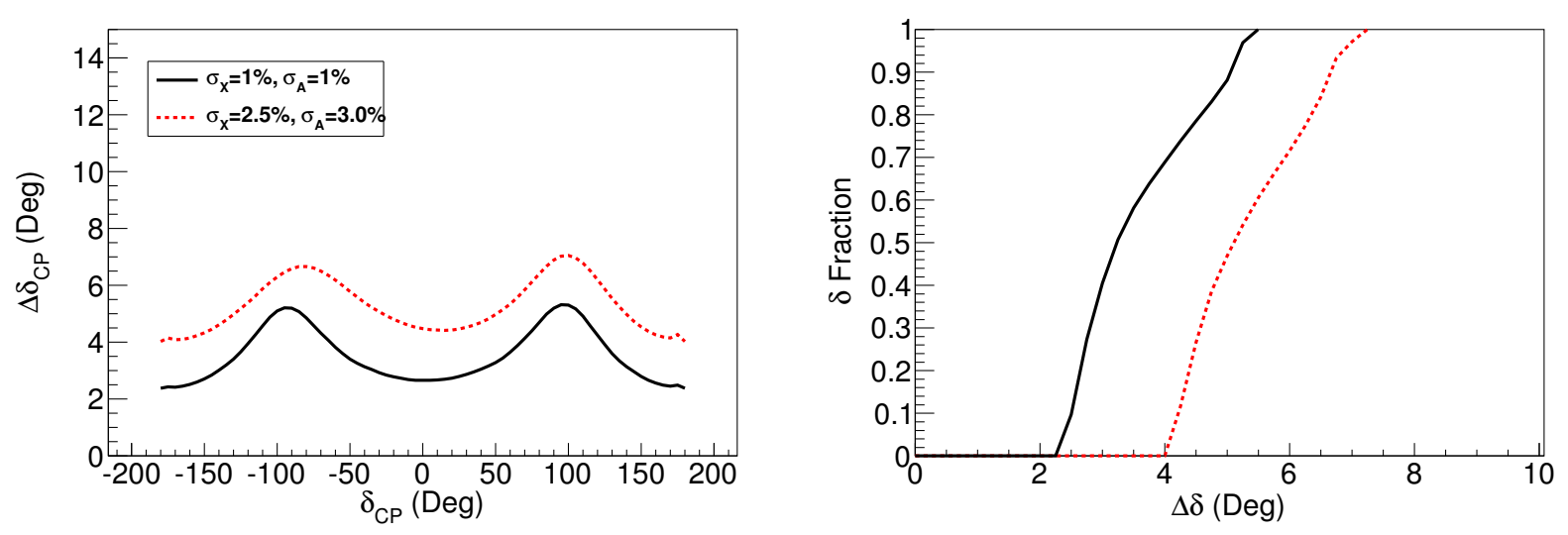

FIG. 26: Left: Precision in the value of $\delta_{C P}$ as a function of $\delta_{C P}$ under two different assumptions: top line (3.0\% normalisation and 2.5\% cross-section error), bottom line (1\% normalisation and cross-section error). Right: $\delta_{C P}$ fraction coverage that can be achieved above each value of $\Delta \delta_{C P}$ for $3.0 \%$ and $2.5 \%$ errors (right curve) and for $1 \%$ and $1 \%$ errors (left curve).

systematic errors: $\left(\sigma_{A}, \sigma_{x}\right)=(3.0 \%, 2.5 \%)$ and $(1.0 \%, 1.0 \%)$. Depending on the value of $\delta_{C P}$ and the level of systematic error, the accuracy in $\Delta \delta_{C P}$ is between $2.5 \%$ and $5 \%$. Figure 26 (right) shows the $\delta$ fraction coverage that can be achieved above the value of $\Delta \delta_{C P}$ determined at a Neutrino Factory for each of the two assumptions about the systematic error.

\section{CONCLUSIONS}

A new GEANT4 simulation of the Magnetised Iron Neutrino Detector (MIND), using GENIE as the neutrino event generator, has been developed to determine the performance of MIND at a Neutrino Factory. Considering the spectrum of neutrino interactions in the energy region 0-25 GeV produced by the GENIE generator, this simulation has been used to study the efficiency and the background rejection of MIND. A detector of 100 ktonne at $2000 \mathrm{~km}$ was used to determine the expected sensitivity to $\delta_{C P}$ and the mass hierarchy at a Neutrino Factory for a value of $\sin ^{2} \theta_{13} \sim 0.1$, as determined recently by reactor and accelerator neutrino oscillation experiments.

The proportions of quasi-elastic, single pion production and deep inelastic events obtained from GENIE have been benchmarked with experiments and a parametrisation of the total 
interaction spectrum, in agreement with data, was used for the simulation. Digitisation of the events tracked through the simulation assumed read-out of the scintillator using WLS fibres and electronics with $30 \% \mathrm{QE}$ and a standard deviation on signal response of $6 \%$. Both assumptions should be achievable and could, perhaps, turn out to be conservative if the current trend in photon detectors and electronics performance continues. These events have been used to study the efficiency and background suppression of MIND in a search for wrong sign muons at a Neutrino Factory storing both muon polarities. A re-optimised reconstruction algorithm and a new analysis applied to data-sets comprising several million $\nu_{\mu}\left(\bar{\nu}_{\mu}\right)$ and $\bar{\nu}_{e}\left(\nu_{e}\right)$ CC and NC events, resulted in response matrices describing the expected response of the detector. MIND showed an efficiency plateau from $5 \mathrm{GeV}$ in true neutrino energy at $\sim 60 \%$ for $\nu_{\mu}$ and $\sim 70 \%$ for $\bar{\nu}_{\mu}$ and thresholds at $\sim 2 \mathrm{GeV}$. All beam inherent backgrounds were simultaneously suppressed at a level around $10^{-4}$. The difference in efficiencies for the two polarities has been studied and found to be predominantly due to the difference in the inelasticity spectrum expected for neutrino and anti-neutrino interactions. We expect the dominant systematic errors to be due to the hadronic energy resolution and due to the different neutrino interaction types. Each contribute an expected systematic error on the signal efficiency of around $1 \%$.

The response of MIND and a parametrisation of the total neutrino and antineutrino crosssections, calculated from the output of the GENIE event generator, were used to study the sensitivity of two MIND detectors at the Neutrino Factory. The obtained measurement of key parameters of the PMNS matrix indicate that such an experiment would determine the mass hierarchy, irrespective of the value of $\delta_{C P}$, at a level better than $5 \sigma$, could perform measurements of $\delta_{C P}$ with an accuracy between $3 \%$ and $5 \%$ and would have an $85 \% \delta_{C P}$ coverage for the currently preferred value of $\theta_{13} \sim 9.0^{\circ}$.

\section{ACKNOWLEDGMENTS}

The authors acknowledge the support of the European Community under the European Commission Framework Programme 7 Design Study: EUROnu, Project Number 212372. The work was supported by the Science and Technology Facilities Council (UK) and by the Spanish Ministry of Education and Science. 
[1] S. Geer, Phys. Rev. D57, 6989 (1998), hep-ph/9712290.

[2] A. Hillairet et al. (TWIST Collaboration), Phys.Rev. D85, 092013 (2012), arXiv:1112.3606 [hep-ex].

[3] A. De Rújula, M. B. Gavela, and P. Hernández, Nucl. Phys. B547, 21 (1999), arXiv:hep$\mathrm{ph} / 9811390$.

[4] A. Cervera et al., Nucl. Phys. B579, 17 (2000), arXiv:hep-ph/0002108.

[5] A. Bandyopadhyay et al. (ISS Physics Working Group), Rept. Prog. Phys. 72, 106201 (2009), arXiv:0710.4947 [hep-ph].

[6] The ISS Accelerator Working Group et al., JINST 4, P07001 (2009).

[7] D. G. Michael et al. (MINOS), Nucl. Instrum. Meth. A596, 190 (2008), arXiv:0805.3170 [physics.ins-det].

[8] A. Cervera, F. Dydak, and J. Gomez Cadenas, Nucl. Instrum. Meth. A451, 123 (2000).

[9] A. Cervera-Villanueva, AIP Conf. Proc. 981, 178 (2008).

[10] T. Abe et al. (ISS Detector Working Group), JINST 4, T05001 (2009), arXiv:0712.4129 [physics.ins-det].

[11] S. K. Agarwalla, P. Huber, J. Tang, and W. Winter, JHEP 1101, 120 (2011), arXiv:1012.1872 [hep-ph],

[12] "The International Design Study for the Neutrino Factory," URL: https://www.idsnf.org/wiki/FrontPage.

[13] "EUROnu: A High Intensity Neutrino Oscillation Facility in Europe," URL: http://www.euronu.org/.

[14] A. Cervera and A. Laing, PoS Nufact08, 042 (2008).

[15] A. Cervera, A. Laing, J. Martin-Albo, and F. J. P. Soler, Nucl. Instrum. Meth. A624, 601 (2010), arXiv:1004.0358 [hep-ex],

[16] C. Andreopoulos et al., Nucl. Instrum. Meth. A614, 87 (2010), arXiv:0905.2517 [hep-ph],

[17] D. Casper, Nucl. Phys. Proc. Suppl. 112, 161 (2002), arXiv:hep-ph/0208030.

[18] S. Agostinelli et al. (GEANT4), Nucl. Instrum. Meth. A506, 250 (2003).

[19] J. Apostolakis and D. H. Wright (Geant4), AIP Conf. Proc. 896, 1 (2007). 
[20] S. Choubey et al. (IDS-NF), (2011), arXiv:1112.2853 [hep-ex],

[21] F. An et al. (DAYA-BAY Collaboration), Phys.Rev.Lett. 108, 171803 (2012), arXiv:1203.1669 $[$ hep-ex].

[22] J. Ahn et al. (RENO collaboration), Phys.Rev.Lett. 108, 191802 (2012), arXiv:1204.0626 [hep-ex].

[23] Y. Abe et al. (DOUBLE-CHOOZ Collaboration), Phys.Rev.Lett. 108, 131801 (2012), arXiv:1112.6353 [hep-ex],

[24] K. Abe et al. (T2K Collaboration), Phys.Rev.Lett. 107, 041801 (2011), arXiv:1106.2822 [hep$\mathrm{ex}]$.

[25] P. Adamson et al. (MINOS Collaboration), Phys.Rev.Lett. 107, 181802 (2011), arXiv:1108.0015 [hep-ex],

[26] D. Indumathi and N. Sinha, Phys. Rev. D80, 113012 (2009), arXiv:0910.2020 [hep-ph].

[27] A. Donini, J. J. Gómez Cadenas, and D. Meloni, JHEP 02, 095 (2011), arXiv:1005.2275 $[$ hep-ph],

[28] G. Ingelman, A. Edin, and J. Rathsman, Computer Physics Communications 101, 108 (1997).

[29] "Geant4 Physics Reference Manual," http://cern.ch/geant4/UserDocumentation/UsersGuides/ PhysicsReferenceManual/html/.

[30] A. Pla-Dalmau, A. D. Bross, V. V. Rykalin, and B. M. Wood (MINERvA), "Extruded plastic scintillator for MINERvA," (2005), proceedings of 2005 IEEE Nuclear Science Symposium and Medical Imaging Conference, El Conquistador Resort, Puerto Rico, 23-29 Oct 2005.

[31] A. Cervera-Villanueva, J. J. Gomez-Cadenas, and J. A. Hernando, Nucl. Instrum. Meth. A534, 180 (2004).

[32] D. Emeliyanov, I. Gorbounov, and I. Kisel, "OTR/ITR-CATS: Tracking Based on Cellular Automaton and Kalman Filter," (2001), HERA-B note 01-137.

[33] A. Laing, PhD thesis, University of Glasgow (2010).

[34] P. Adamson et al., Nucl. Instrum. Meth. A556, 119 (2006).

[35] G. Bari et al., Nucl. Instrum. Meth. A508, 170 (2003).

[36] A. Blondel, M. Campanelli, and M. Fechner, Nucl. Instrum. Meth. A535, 665 (2004).

[37] P. Adamson et al. (MINOS), Phys. Rev. D 77, 072002 (2008), arXiv:0711.0769 [hep-ex]

[38] A. Cervera-Villanueva, Nucl. Phys. Proc. Suppl. 149, 201 (2005).

[39] K. Zuber, Neutrino physics (IOP, 2003). 
[40] T. Schwetz, M. A. Tortola, and J. W. F. Valle, New J. Phys. 10, 113011 (2008), arXiv:0808.2016 [hep-ph].

[41] A. M. Dziewonski and D. L. Anderson, Phys. Earth Planet. Interiors 25, 297 (1981).

[42] V. Lyubushkin et al. (NOMAD), Eur. Phys. J. C63, 355 (2009), arXiv:0812.4543 [hep-ex].

[43] A. A. Aguilar-Arevalo et al. (MiniBooNE), Phys. Rev. D81, 092005 (2010), arXiv:1002.2680.

[44] A. Rodriguez et al. (K2K), Phys. Rev. D78, 032003 (2008), arXiv:0805.0186 [hep-ex].

[45] J. Burguet-Castell, M. B. Gavela, J. J. Gómez-Cadenas, P. Hernández, and O. Mena, Nucl. Phys. B608, 301 (2001), arXiv:hep-ph/0103258.

[46] J. Burguet-Castell, M. B. Gavela, J. J. Gómez-Cadenas, P. Hernández, and O. Mena, Nucl. Phys. B646, 301 (2002), arXiv:hep-ph/0207080.

[47] J. Burguet-Castell, D. Casper, E. Couce, J. J. Gómez-Cadenas, and P. Hernández, Nucl. Phys. B725, 306 (2005), arXiv:hep-ph/0503021.

[48] P. Coloma, A. Donini, E. Fernandez-Martinez, and P. Hernandez, JHEP 1206, 073 (2012), arXiv:1203.5651 [hep-ph]. 


\section{APPENDIX}

This appendix summarizes the response matrices of signal (wrong sign $\nu_{\mu}$ and $\bar{\nu}_{\mu}$ appearance) and all backgrounds. ( $\bar{\nu}_{\mu}$ and $\nu_{\mu} \mathrm{CC}, \bar{\nu}_{e}$ and $\nu_{e} \mathrm{CC}, \bar{\nu}_{\tau}$ and $\nu_{\tau} \mathrm{CC}$, and NC) in bins of true and reconstructed neutrino energy relevant to an oscillation analysis. Each entry in the table is the survival probability for each species. In all tables, columns represent the true neutrino energy in $\mathrm{GeV}$ and rows the reconstructed energy, also in GeV. The overflow bin in reconstructed energy represents all events with a reconstructed energy greater than the known maximum.

\section{A. $\nu_{\mu}$ appearance}

\begin{tabular}{|c||c|c|c|c|c|c|c|c|c|c|}
\hline & $0.0-1.0$ & $1.0-2.0$ & $2.0-3.0$ & $3.0-4.0$ & $4.0-5.0$ & $5.0-6.0$ & $6.0-7.0$ & $7.0-8.0$ & $8.0-9.0$ & $9.0-10.0$ \\
\hline $0.0-1.0$ & 0 & 1.392 & 3.940 & 2.052 & 2.402 & 1.323 & 0.3669 & 0.2460 & 0.3710 & 0.1218 \\
$1.0-2.0$ & 0 & 222.0 & 243.6 & 45.87 & 28.82 & 21.65 & 9.660 & 6.519 & 1.608 & 2.679 \\
$2.0-3.0$ & 0 & 149.4 & 1238 & 568.5 & 144.5 & 68.80 & 34.73 & 22.63 & 14.10 & 7.551 \\
$3.0-4.0$ & 0 & 14.13 & 807.9 & 1790 & 813.2 & 270.8 & 110.4 & 60.52 & 41.31 & 26.79 \\
$4.0-5.0$ & 0 & 1.392 & 162.0 & 1340 & 1848 & 859.0 & 318.7 & 146.9 & 81.87 & 57.24 \\
$5.0-6.0$ & 0 & 0.1070 & 14.64 & 375.5 & 1516 & 1695 & 805.7 & 339.7 & 152.2 & 83.54 \\
$6.0-7.0$ & 0 & 0.1070 & 1.914 & 62.40 & 593.3 & 1630 & 1616 & 841.6 & 369.3 & 178.8 \\
$7.0-8.0$ & 0 & 0 & 0.3378 & 7.725 & 123.1 & 744.8 & 1563 & 1462 & 783.8 & 347.1 \\
$8.0-9.0$ & 0 & 0 & 0 & 0.7242 & 18.37 & 200.4 & 836.3 & 1547 & 1360 & 771.6 \\
$9.0-10.0$ & 0 & 0 & 0.1126 & 0.3621 & 2.402 & 35.24 & 277.6 & 891.3 & 1454 & 1289 \\
$10.0-11.0$ & 0 & 0 & 0 & 0 & 0.6004 & 8.059 & 83.52 & 474.8 & 1520 & 3014 \\
\hline
\end{tabular}

TABLE III: Golden channel $\nu_{\mu}$ appearance signal efficiency. All values $\times 10^{-4}$. 


\begin{tabular}{|c||c|c|c|c|c|c|c|c|c|c|}
\hline & $0.0-1.0$ & $1.0-2.0$ & $2.0-3.0$ & $3.0-4.0$ & $4.0-5.0$ & $5.0-6.0$ & $6.0-7.0$ & $7.0-8.0$ & $8.0-9.0$ & $9.0-10.0$ \\
\hline $0.0-1.0$ & 0 & 0 & 0 & 0 & 0 & 0 & 0 & 0 & 0 & 0 \\
$1.0-2.0$ & 0 & 0 & 0 & 0 & 0 & 0 & 0 & 0 & 0 & 0 \\
$2.0-3.0$ & 0 & 0 & 0 & 0 & 0 & 0.1203 & 0.1218 & 0 & 0 & 0.1198 \\
$3.0-4.0$ & 0 & 0 & 0 & 0 & 0 & 0.2406 & 0 & 0 & 0.1220 & 0.1198 \\
$4.0-5.0$ & 0 & 0 & 0 & 0 & 0 & 0 & 0.2437 & 0.2439 & 0 & 0.1198 \\
$5.0-6.0$ & 0 & 0 & 0 & 0 & 0 & 0 & 0 & 0 & 0.1220 & 0 \\
$6.0-7.0$ & 0 & 0 & 0 & 0 & 0 & 0 & 0 & 0.1220 & 0.1220 & 0.2396 \\
$7.0-8.0$ & 0 & 0 & 0 & 0 & 0 & 0.1203 & 0 & 0.1220 & 0 & 0.2396 \\
$8.0-9.0$ & 0 & 0 & 0 & 0 & 0 & 0 & 0 & 0 & 0 & 0.1198 \\
$9.0-10.0$ & 0 & 0 & 0 & 0 & 0 & 0 & 0.1218 & 0 & 0 & 0 \\
$10.0-11.0$ & 0 & 0 & 0 & 0 & 0 & 0 & 0 & 0 & 0 & 0.1198 \\
\hline
\end{tabular}

TABLE IV: $\mu^{-}$background from charge mis-identified $\bar{\nu}_{\mu}$ CC events All values $\times 10^{-4}$.

\begin{tabular}{|c||c|c|c|c|c|c|c|c|c|c|}
\hline & $0.0-1.0$ & $1.0-2.0$ & $2.0-3.0$ & $3.0-4.0$ & $4.0-5.0$ & $5.0-6.0$ & $6.0-7.0$ & $7.0-8.0$ & $8.0-9.0$ & $9.0-10.0$ \\
\hline $0.0-1.0$ & 0 & 0 & 0 & 0 & 0 & 0 & 0 & 0 & 0 & 0 \\
$1.0-2.0$ & 0 & 0 & 0 & 0 & 0 & 0 & 0 & 0 & 0 & 0 \\
$2.0-3.0$ & 0 & 0 & 0 & 0 & 0 & 0 & 0 & 0 & 0 & 0 \\
$3.0-4.0$ & 0 & 0 & 0 & 0 & 0.0596 & 0 & 0 & 0 & 0 & 0 \\
$4.0-5.0$ & 0 & 0 & 0 & 0.0596 & 0 & 0 & 0.0614 & 0 & 0 & 0 \\
$5.0-6.0$ & 0 & 0 & 0 & 0 & 0 & 0 & 0 & 0 & 0 & 0.0610 \\
$6.0-7.0$ & 0 & 0 & 0 & 0 & 0 & 0 & 0 & 0 & 0 & 0 \\
$7.0-8.0$ & 0 & 0 & 0 & 0 & 0 & 0 & 0 & 0 & 0 & 0 \\
$8.0-9.0$ & 0 & 0 & 0 & 0 & 0 & 0 & 0 & 0 & 0 & 0 \\
$9.0-10.0$ & 0 & 0 & 0 & 0 & 0 & 0 & 0 & 0 & 0 & 0 \\
$10.0-11.0$ & 0 & 0 & 0 & 0 & 0 & 0 & 0 & 0 & 0 & 0.0610 \\
\hline
\end{tabular}

TABLE V: $\mu^{-}$background from $\nu_{e}$ CC events. All values $\times 10^{-4}$. 


\begin{tabular}{|c||c|c|c|c|c|c|c|c|c|c|}
\hline & $0.0-1.0$ & $1.0-2.0$ & $2.0-3.0$ & $3.0-4.0$ & $4.0-5.0$ & $5.0-6.0$ & $6.0-7.0$ & $7.0-8.0$ & $8.0-9.0$ & $9.0-10.0$ \\
\hline $0.0-1.0$ & 0 & 0 & 0 & 0 & 0 & 0 & 0 & 0 & 0 & 0 \\
$1.0-2.0$ & 0 & 0 & 0 & 0.1729 & 0.0581 & 0.3538 & 0.0602 & 0.1221 & 0 & 0.0612 \\
$2.0-3.0$ & 0 & 0 & 0.1068 & 0 & 0.3488 & 0.2359 & 0.1807 & 0.4272 & 0.3680 & 0.1224 \\
$3.0-4.0$ & 0 & 0 & 0 & 0.2305 & 0.8138 & 0.8844 & 0.8431 & 0.6713 & 0.6133 & 0.8566 \\
$4.0-5.0$ & 0 & 0 & 0 & 0.1152 & 0.5232 & 0.7076 & 1.144 & 1.098 & 0.9812 & 0.9178 \\
$5.0-6.0$ & 0 & 0 & 0 & 0.0576 & 0.0581 & 0.5307 & 0.3011 & 0.5492 & 0.6746 & 0.6119 \\
$6.0-7.0$ & 0 & 0 & 0 & 0 & 0 & 0.1769 & 0.3011 & 0.4272 & 0.5519 & 0.4895 \\
$7.0-8.0$ & 0 & 0 & 0 & 0 & 0 & 0 & 0.0602 & 0.4272 & 0.2453 & 0.4283 \\
$8.0-9.0$ & 0 & 0 & 0 & 0 & 0 & 0 & 0 & 0.0610 & 0.1840 & 0.4283 \\
$9.0-10.0$ & 0 & 0 & 0 & 0 & 0 & 0 & 0 & 0 & 0 & 0.1836 \\
$10.0-11.0$ & 0 & 0 & 0 & 0 & 0 & 0 & 0 & 0 & 0 & 0.1836 \\
\hline
\end{tabular}

TABLE VI: $\mu^{-}$background from $\bar{\nu}_{\mu} \mathrm{NC}$ events. All values $\times 10^{-4}$.

\begin{tabular}{|c||c|c|c|c|c|c|c|c|c|c|}
\hline & $0.0-1.0$ & $1.0-2.0$ & $2.0-3.0$ & $3.0-4.0$ & $4.0-5.0$ & $5.0-6.0$ & $6.0-7.0$ & $7.0-8.0$ & $8.0-9.0$ & $9.0-10.0$ \\
\hline $0.0-1.0$ & 0 & 0 & 0 & 0 & 0.5833 & 0 & 0 & 0.3747 & 0 & 0.1426 \\
$1.0-2.0$ & 0 & 0 & 0 & 14.70 & 8.750 & 8.569 & 12.33 & 8.992 & 9.264 & 6.845 \\
$2.0-3.0$ & 0 & 0 & 0 & 49.97 & 51.33 & 54.27 & 65.98 & 67.44 & 52.33 & 45.35 \\
$3.0-4.0$ & 0 & 0 & 0 & 38.21 & 51.92 & 58.08 & 71.91 & 76.06 & 78.66 & 79.71 \\
$4.0-5.0$ & 0 & 0 & 0 & 11.76 & 18.08 & 38.08 & 47.49 & 57.32 & 61.27 & 70.16 \\
$5.0-6.0$ & 0 & 0 & 0 & 0 & 5.833 & 16.19 & 28.77 & 32.41 & 33.32 & 42.78 \\
$6.0-7.0$ & 0 & 0 & 0 & 0 & 0 & 3.808 & 12.33 & 17.23 & 18.69 & 25.67 \\
$7.0-8.0$ & 0 & 0 & 0 & 0 & 0 & 1.587 & 4.109 & 10.30 & 10.89 & 13.69 \\
$8.0-9.0$ & 0 & 0 & 0 & 0 & 0 & 0 & 1.142 & 2.810 & 5.526 & 7.986 \\
$9.0-10.0$ & 0 & 0 & 0 & 0 & 0 & 0 & 0.2283 & 0.3747 & 2.113 & 4.278 \\
$10.0-11.0$ & 0 & 0 & 0 & 0 & 0 & 0 & 0 & 0 & 1.788 & 2.139 \\
\hline
\end{tabular}

TABLE VII: $\mu^{-}$reconstructed from $\nu_{\tau}$ CC events. All values $\times 10^{-4}$. 


\begin{tabular}{|c||c|c|c|c|c|c|c|c|c|c|}
\hline & $0.0-1.0$ & $1.0-2.0$ & $2.0-3.0$ & $3.0-4.0$ & $4.0-5.0$ & $5.0-6.0$ & $6.0-7.0$ & $7.0-8.0$ & $8.0-9.0$ & $9.0-10.0$ \\
\hline $0.0-1.0$ & 0 & 0 & 0 & 0 & 0 & 0 & 0 & 0 & 0 & 0 \\
$1.0-2.0$ & 0 & 0 & 0 & 0 & 0 & 0 & 0 & 0 & 0 & 0 \\
$2.0-3.0$ & 0 & 0 & 0 & 0 & 0 & 0 & 0 & 0 & 0 & 0 \\
$3.0-4.0$ & 0 & 0 & 0 & 0 & 0 & 0 & 0 & 0.1637 & 0.4287 & 0 \\
$4.0-5.0$ & 0 & 0 & 0 & 0 & 0 & 0 & 0 & 0.1637 & 0.2858 & 0 \\
$5.0-6.0$ & 0 & 0 & 0 & 0 & 0 & 0 & 0 & 0 & 0 & 0 \\
$6.0-7.0$ & 0 & 0 & 0 & 0 & 0 & 0 & 0 & 0 & 0 & 0 \\
$7.0-8.0$ & 0 & 0 & 0 & 0 & 0 & 0 & 0 & 0 & 0 & 0 \\
$8.0-9.0$ & 0 & 0 & 0 & 0 & 0 & 0 & 0 & 0 & 0 & 0 \\
$9.0-10.0$ & 0 & 0 & 0 & 0 & 0 & 0 & 0 & 0 & 0 & 0 \\
$10.0-11.0$ & 0 & 0 & 0 & 0 & 0 & 0 & 0 & 0 & 0 & 0 \\
\hline
\end{tabular}

TABLE VIII: $\mu^{-}$background from $\bar{\nu}_{\tau}$ CC events. All values $\times 10^{-4}$.

\section{B. $\bar{\nu}_{\mu}$ appearance}

\begin{tabular}{|c||c|c|c|c|c|c|c|c|c|c|}
\hline & $0.0-1.0$ & $1.0-2.0$ & $2.0-3.0$ & $3.0-4.0$ & $4.0-5.0$ & $5.0-6.0$ & $6.0-7.0$ & $7.0-8.0$ & $8.0-9.0$ & $9.0-10.0$ \\
\hline $0.0-1.0$ & 0 & 4.606 & 22.28 & 14.26 & 9.296 & 3.729 & 1.340 & 0.3659 & 0 & 0.7188 \\
$1.0-2.0$ & 0 & 85.70 & 262.9 & 93.41 & 54.43 & 26.11 & 18.15 & 9.025 & 4.757 & 1.318 \\
$2.0-3.0$ & 0 & 84.87 & 1565 & 656.4 & 116.1 & 51.85 & 39.48 & 28.05 & 20.61 & 12.94 \\
$3.0-4.0$ & 0 & 5.304 & 990.1 & 2364 & 795.4 & 164.5 & 63.85 & 49.15 & 46.11 & 38.57 \\
$4.0-5.0$ & 0 & 0.1396 & 161.9 & 1904 & 2513 & 880.2 & 226.6 & 89.76 & 66.35 & 54.63 \\
$5.0-6.0$ & 0 & 0 & 10.52 & 477.7 & 2145 & 2218 & 851.9 & 246.6 & 97.94 & 64.45 \\
$6.0-7.0$ & 0 & 0 & 0 & 60.02 & 801.6 & 2221 & 2032 & 842.4 & 283.0 & 111.5 \\
$7.0-8.0$ & 0 & 0 & 0.1238 & 3.751 & 152.5 & 1022 & 2181 & 1896 & 840.2 & 307.4 \\
$8.0-9.0$ & 0 & 0 & 0 & 0.6252 & 19.33 & 263.2 & 1169 & 2108 & 1738 & 832.3 \\
$9.0-10.0$ & 0 & 0 & 0 & 0.1250 & 1.223 & 47.28 & 355.9 & 1230 & 1996 & 1633 \\
$10.0-11.0$ & 0 & 0 & 0 & 0.1250 & 0.3670 & 8.301 & 98.33 & 603.4 & 2024 & 4033 \\
\hline
\end{tabular}

TABLE IX: Golden channel $\bar{\nu}_{\mu}$ appearance signal efficiency. All values $\times 10^{-4}$. 


\begin{tabular}{|c||c|c|c|c|c|c|c|c|c|c|}
\hline & $0.0-1.0$ & $1.0-2.0$ & $2.0-3.0$ & $3.0-4.0$ & $4.0-5.0$ & $5.0-6.0$ & $6.0-7.0$ & $7.0-8.0$ & $8.0-9.0$ & $9.0-10.0$ \\
\hline $0.0-1.0$ & 0 & 0 & 0 & 0 & 0 & 0 & 0 & 0 & 0 & 0 \\
$1.0-2.0$ & 0 & 0 & 0 & 0 & 0 & 0 & 0 & 0 & 0 & 0 \\
$2.0-3.0$ & 0 & 0 & 0 & 0 & 0 & 0 & 0 & 0 & 0 & 0 \\
$3.0-4.0$ & 0 & 0 & 0.3378 & 0.6035 & 0 & 0.2406 & 0.1223 & 0.1230 & 0 & 0 \\
$4.0-5.0$ & 0 & 0 & 0.2252 & 0.8449 & 0.3602 & 0.2406 & 0 & 0 & 0 & 0.2436 \\
$5.0-6.0$ & 0 & 0 & 0 & 0.1207 & 0.2402 & 0 & 0.1223 & 0.3690 & 0.1237 & 0.1218 \\
$6.0-7.0$ & 0 & 0 & 0 & 0 & 0 & 0.1203 & 0.2446 & 0 & 0.1237 & 0 \\
$7.0-8.0$ & 0 & 0 & 0 & 0 & 0 & 0.2406 & 0.1223 & 0.1230 & 0 & 0 \\
$8.0-9.0$ & 0 & 0 & 0 & 0.1207 & 0 & 0 & 0 & 0 & 0 & 0 \\
$9.0-10.0$ & 0 & 0 & 0 & 0 & 0 & 0 & 0 & 0.1230 & 0.1237 & 0.1218 \\
$10.0-11.0$ & 0 & 0 & 0 & 0 & 0 & 0.1203 & 0.2446 & 0.2460 & 0 & 0.3654 \\
\hline
\end{tabular}

TABLE X: $\mu^{+}$background from charge mis-identified $\nu_{\mu}$ CC events All values $\times 10^{-4}$.

\begin{tabular}{|c||c|c|c|c|c|c|c|c|c|c|}
\hline & $0.0-1.0$ & $1.0-2.0$ & $2.0-3.0$ & $3.0-4.0$ & $4.0-5.0$ & $5.0-6.0$ & $6.0-7.0$ & $7.0-8.0$ & $8.0-9.0$ & $9.0-10.0$ \\
\hline $0.0-1.0$ & 0 & 0 & 0 & 0 & 0 & 0 & 0 & 0 & 0 & 0 \\
$1.0-2.0$ & 0 & 0 & 0 & 0 & 0 & 0 & 0 & 0 & 0 & 0 \\
$2.0-3.0$ & 0 & 0 & 0 & 0 & 0 & 0.0605 & 0.0608 & 0 & 0 & 0 \\
$3.0-4.0$ & 0 & 0 & 0.0613 & 0 & 0.0609 & 0 & 0 & 0.1221 & 0.1215 & 0.0602 \\
$4.0-5.0$ & 0 & 0 & 0 & 0.0627 & 0.1218 & 0.1211 & 0.2431 & 0.1832 & 0.1215 & 0.1204 \\
$5.0-6.0$ & 0 & 0 & 0 & 0 & 0 & 0 & 0 & 0.0611 & 0 & 0 \\
$6.0-7.0$ & 0 & 0 & 0 & 0 & 0.0609 & 0 & 0 & 0.1221 & 0 & 0 \\
$7.0-8.0$ & 0 & 0 & 0 & 0 & 0 & 0 & 0 & 0 & 0 & 0 \\
$8.0-9.0$ & 0 & 0 & 0 & 0 & 0 & 0 & 0 & 0 & 0 & 0.0602 \\
$9.0-10.0$ & 0 & 0 & 0 & 0 & 0 & 0 & 0 & 0.0611 & 0.0608 & 0.0602 \\
$10.0-11.0$ & 0 & 0 & 0 & 0 & 0 & 0 & 0 & 0 & 0 & 0 \\
\hline
\end{tabular}

TABLE XI: $\mu^{+}$background from $\bar{\nu}_{e}$ CC events. All values $\times 10^{-4}$. 


\begin{tabular}{|c||c|c|c|c|c|c|c|c|c|c|}
\hline & $0.0-1.0$ & $1.0-2.0$ & $2.0-3.0$ & $3.0-4.0$ & $4.0-5.0$ & $5.0-6.0$ & $6.0-7.0$ & $7.0-8.0$ & $8.0-9.0$ & $9.0-10.0$ \\
\hline $0.0-1.0$ & 0 & 0 & 0 & 0 & 0 & 0 & 0 & 0 & 0 & 0 \\
$1.0-2.0$ & 0 & 0 & 0 & 0 & 0 & 0.0586 & 0 & 0.0607 & 0 & 0 \\
$2.0-3.0$ & 0 & 0 & 0 & 0.1167 & 0 & 0.1173 & 0 & 0.0607 & 0 & 0.0606 \\
$3.0-4.0$ & 0 & 0 & 0 & 0 & 0 & 0.1759 & 0.0597 & 0.1213 & 0.1210 & 0.2422 \\
$4.0-5.0$ & 0 & 0 & 0 & 0 & 0 & 0.0586 & 0.1194 & 0.2427 & 0.1814 & 0.0606 \\
$5.0-6.0$ & 0 & 0 & 0 & 0 & 0 & 0 & 0.0597 & 0.0607 & 0.1210 & 0.1817 \\
$6.0-7.0$ & 0 & 0 & 0 & 0 & 0 & 0 & 0 & 0.0607 & 0.1814 & 0.0606 \\
$7.0-8.0$ & 0 & 0 & 0 & 0 & 0 & 0 & 0 & 0 & 0.0605 & 0 \\
$8.0-9.0$ & 0 & 0 & 0 & 0 & 0 & 0 & 0 & 0 & 0 & 0 \\
$9.0-10.0$ & 0 & 0 & 0 & 0 & 0 & 0 & 0 & 0 & 0 & 0.0606 \\
$10.0-11.0$ & 0 & 0 & 0 & 0 & 0 & 0 & 0 & 0 & 0 & 0 \\
\hline
\end{tabular}

TABLE XII: $\mu^{+}$background from $\nu_{\mu} \mathrm{NC}$ events. All values $\times 10^{-4}$.

\begin{tabular}{|c||c|c|c|c|c|c|c|c|c|c|}
\hline & $0.0-1.0$ & $1.0-2.0$ & $2.0-3.0$ & $3.0-4.0$ & $4.0-5.0$ & $5.0-6.0$ & $6.0-7.0$ & $7.0-8.0$ & $8.0-9.0$ & $9.0-10.0$ \\
\hline $0.0-1.0$ & 0 & 0 & 0 & 15.53 & 1.498 & 2.763 & 3.066 & 2.128 & 1.857 & 2.020 \\
$1.0-2.0$ & 0 & 0 & 0 & 0 & 22.47 & 33.16 & 31.88 & 27.66 & 25.29 & 23.99 \\
$2.0-3.0$ & 0 & 0 & 0 & 62.11 & 64.41 & 77.99 & 74.19 & 75.28 & 67.44 & 59.34 \\
$3.0-4.0$ & 0 & 0 & 0 & 54.35 & 63.67 & 68.47 & 66.62 & 61.70 & 73.59 & 69.82 \\
$4.0-5.0$ & 0 & 0 & 0 & 15.53 & 28.46 & 42.99 & 54.36 & 55.15 & 55.15 & 53.91 \\
$5.0-6.0$ & 0 & 0 & 0 & 0 & 5.243 & 19.65 & 36.17 & 36.99 & 41.29 & 43.43 \\
$6.0-7.0$ & 0 & 0 & 0 & 0 & 3.745 & 5.220 & 11.44 & 20.62 & 27.01 & 26.39 \\
$7.0-8.0$ & 0 & 0 & 0 & 0 & 0 & 0.6141 & 4.292 & 12.27 & 16.72 & 18.94 \\
$8.0-9.0$ & 0 & 0 & 0 & 0 & 0 & 0 & 1.635 & 2.619 & 5.430 & 10.48 \\
$9.0-10.0$ & 0 & 0 & 0 & 0 & 0 & 0 & 0.4087 & 1.964 & 3.572 & 5.555 \\
$10.0-11.0$ & 0 & 0 & 0 & 0 & 0 & 0.3070 & 0.2044 & 0.4910 & 1.286 & 5.176 \\
\hline
\end{tabular}

TABLE XIII: $\mu^{+}$reconstructed from $\bar{\nu}_{\tau}$ CC events. All values $\times 10^{-4}$. 


\begin{tabular}{|c||c|c|c|c|c|c|c|c|c|c|}
\hline & $0.0-1.0$ & $1.0-2.0$ & $2.0-3.0$ & $3.0-4.0$ & $4.0-5.0$ & $5.0-6.0$ & $6.0-7.0$ & $7.0-8.0$ & $8.0-9.0$ & $9.0-10.0$ \\
\hline $0.0-1.0$ & 0 & 0 & 0 & 0 & 0 & 0 & 0 & 0 & 0 & 0 \\
$1.0-2.0$ & 0 & 0 & 0 & 0 & 0 & 0 & 0.6849 & 0.1873 & 0.6501 & 0.2852 \\
$2.0-3.0$ & 0 & 0 & 0 & 0 & 0 & 0.3174 & 0.2283 & 0.9367 & 0.4876 & 0.9982 \\
$3.0-4.0$ & 0 & 0 & 0 & 0 & 0.5833 & 0 & 0.2283 & 0.3747 & 0.9751 & 1.426 \\
$4.0-5.0$ & 0 & 0 & 0 & 0 & 0 & 0 & 0 & 0.3747 & 0.4876 & 0.7130 \\
$5.0-6.0$ & 0 & 0 & 0 & 0 & 0 & 0 & 0 & 0 & 0.3250 & 0.8556 \\
$6.0-7.0$ & 0 & 0 & 0 & 0 & 0 & 0 & 0 & 0 & 0 & 0.1426 \\
$7.0-8.0$ & 0 & 0 & 0 & 0 & 0 & 0 & 0 & 0 & 0 & 0 \\
$8.0-9.0$ & 0 & 0 & 0 & 0 & 0 & 0 & 0 & 0 & 0 & 0 \\
$9.0-10.0$ & 0 & 0 & 0 & 0 & 0 & 0 & 0 & 0 & 0 & 0 \\
$10.0-11.0$ & 0 & 0 & 0 & 0 & 0 & 0 & 0 & 0 & 0 & 0 \\
\hline
\end{tabular}

TABLE XIV: $\mu^{+}$background from $\nu_{\tau}$ CC events. All values $\times 10^{-4}$. 